\title{
Radial gradients in initial mass function sensitive absorption features in the Coma brightest cluster galaxies
}

\author{
Simon Zieleniewski ${ }^{1 \star}$ Ryan C. W. Houghton ${ }^{1}$ Niranjan Thatte $^{1}$ Roger L. Davies ${ }^{1}$ \\ Sam P. Vaughan ${ }^{1}$ \\ ${ }^{1}$ Astrophysics, Denys Wilkinson Building, Keble Road, Oxford, OX1 $4 R H$
}

Submitted $15-10-16$

\begin{abstract}
Using the Oxford Short Wavelength Integral Field specTrograph (SWIFT), we trace radial variations of initial mass function (IMF) sensitive absorption features of three galaxies in the Coma cluster. We obtain resolved spectroscopy of the central $5 \mathrm{kpc}$ for the two central brightest-cluster galaxies (BCGs) NGC4889, NGC4874, and the BCG in the south-west group NGC4839, as well as unresolved data for NGC4873 as a low- $\sigma_{*}$ control. We present radial measurements of the IMF-sensitive features sodium $\mathrm{NaI}_{\text {SDSS }}$, calcium triplet $\mathrm{CaT}$ and iron-hydride FeH0.99, along with the magnesium MgI0.88 and titanium oxide TiO0.89 features. We employ two separate methods for both telluric correction and sky-subtraction around the faint FeH feature to verify our analysis. Within NGC4889 we find strong gradients of NaI $\mathrm{IDSS}_{\text {and }} \mathrm{CaT}$ but a flat $\mathrm{FeH}$ profile, which from comparing to stellar population synthesis models, suggests an old, $\alpha$-enhanced population with a Chabrier, or even bottom-light IMF. The age and abundance is in line with previous studies but the normal IMF is in contrast to recent results suggesting an increased IMF slope with increased velocity dispersion. We measure flat $\mathrm{NaI}_{\text {SDSS }}$ and $\mathrm{FeH}$ profiles within NGC4874 and determine an old, possibly slightly $\alpha$-enhanced and Chabrier IMF population. We find an $\alpha$-enhanced, Chabrier IMF population in NGC4873. Within NGC4839 we measure both strong NaI SDSS $_{\text {and }}$ strong $\mathrm{FeH}$, although with a large systematic uncertainty, suggesting a possible heavier IMF. The IMFs we infer for these galaxies are supported by published dynamical modelling. We stress that IMF constraints should be corroborated by further spectral coverage and independent methods on a galaxy-by-galaxy basis.
\end{abstract}

Key words: galaxies - galaxies: stellar content - galaxies: abundances

\section{INTRODUCTION}

The formation of the stellar content in early type galaxies (ETGs), quantified by the initial mass function (IMF), has recently been the focus of much study. The IMF sets the mass distribution of stars and affects the total mass-to-light ratio of a galaxy, as well as feedback scales from supernovae and chemical enrichment of the interstellar medium. The most direct method to constrain the IMF is through star counts, which makes measuring the IMF an immense challenge for any galaxy other than our own. The IMF in the Milky Way has been constrained by several studies, notably Salpeter (1955), who devised the classical power law form of the IMF, $\xi(m)=k m^{-x}$, where the exponent takes a value of $x=2.35$, down to masses of $\sim 1 \mathrm{M}_{\odot}$. Later studies by Miller \& Scalo (1979); Kroupa (2001) and Chabrier (2003)

* E-mail: simon.zieleniewski@physics.ox.ac.uk incorporated a turn-off at masses below $\sim 1 \mathrm{M}_{\odot}$. Constraining the low-mass end of the IMF is important as these stars dominate the mass budget of galaxies, so changes in quantity can alter the galaxy mass-to-light $(M / L)$ ratio significantly.

Historically, the form of the IMF has generally been assumed to be universal between galaxies. However, recent studies have compounded the notion that the low-mass end of the IMF in fact varies as a function of galaxy properties. Several independent methods have been employed to undertake this work. Dynamical modelling of galaxy kinematics through integral field spectroscopic observations have shown that the mass 'normalisation' (the $M / L$ ) of galaxies systematically increases with galaxy velocity dispersion (Cappellari et al. 2012, 2013b). Strong gravitational lensing results have also suggested M/L variation in ETGs (e.g. Treu et al. 2010; Smith et al. 2015a). However, both of these methods only probe the total mass and so suffer degeneracies between dark and luminous matter components. 
In the absence of direct star counts, the most direct method to probe the stellar content of ETGs is to analyse their integrated stellar spectra. Several gravity-sensitive stellar absorption features are well documented, which vary in strength between dwarf and giant stars. The three key features in the far red region are sodium NaI $\lambda 8190$ (Faber \& French 1980, Schiavon et al. 1997a); the calcium triplet CaT (Cenarro et al. 2001, 2003); and the Wing-Ford band FeH $\lambda 9916$ (Wing \& Ford 1969, Carter et al. 1986, Schiavon et al. 1997b, Cushing et al. 2003). IMF-sensitive features in the optical have also recently been utilised, including titanium oxide TiO features (Spiniello et al. 2012; Ferreras et al. 2013; La Barbera et al. 2013) and calcium monohydride $\mathrm{CaH}$ (Spiniello et al. 2014).

Alongside improvements in instrumentation that have allowed the detection of faint far red features, advances in stellar population modelling over recent years have also majorly contributed to our understanding of the IMF (see e.g. Conroy 2013). Stellar population synthesis (SPS) models now produce simple stellar population (SSP) spectra for varying IMFs (e.g. Vazdekis et al. 2012; Conroy \& van Dokkum 2012a), as well as individual elemental abundance variations (Conroy \& van Dokkum 2012a).

Stellar population gradients within galaxies are now being routinely investigated, greatly aided by advances in integral field spectroscopy (e.g. Kuntschner et al. 2010; Loubser \& Sánchez-Blázquez 2012; Greene et al. 2015; OlivaAltamirano et al. 2015). Recent work by several groups has explored the possibility of radial gradients of IMF-sensitive spectral features in galaxies. Results by Martín-Navarro et al. (2015a) have indicated a gradient to the IMF slope in a massive ETG with a large central velocity dispersion, ranging from very bottom-heavy in the central region to around Salpeter further out. Martín-Navarro et al. (2015b) have also presented results indicating a massive galaxy with a constant bottom-heavy IMF independent of radius.

While these results are important and can be interpreted as a larger fraction of low-mass stars in the central, densest regions of the most massive galaxies, they are based on absorption features which are susceptible to influence from non-IMF factors. In particular they do not utilise the FeH feature, which was used by van Dokkum \& Conroy (2010, 2012); Conroy \& van Dokkum (2012b) in their studies that reignited this field of research. Zieleniewski et al. (2015) and McConnell et al. (2016) have both presented radial measurements of $\mathrm{NaI}, \mathrm{CaT}$ and $\mathrm{FeH}$ in several galaxies: M31 and M32 in Zieleniewski et al. and two massive ETGs in McConnell et al.. Both sets of results showed strong gradients in $\mathrm{NaI}$ but flat profiles (or slightly negative gradients in the case of the ETGs) in FeH. These resulted in discrepant predictions of the IMF between the individual features and were interpreted as normal IMFs with strong gradients in $\mathrm{Na}$ abundance. La Barbera et al. (2016) have also measured radial measurements of $\mathrm{FeH}$ and optical $\mathrm{TiO}$ features in a single massive ETG. They also found a flat FeH profile contrasted with strong $\mathrm{TiO}$ gradients and excluded a bottomheavy $x=3$ IMF, instead invoking a bimodal form with a taper below $1 \mathrm{M}_{\odot}$.

In this paper we add to the small but growing amount of literature regarding radial gradients in IMF-sensitive stellar absorption features of massive ETGs, by presenting results from four galaxies in the Coma cluster. We tar- get the two massive, slow-rotator, brightest cluster galaxies (BCGs) within the main cluster: NGC4889 and NGC4874 $\left(\epsilon=0.36, \lambda_{\mathrm{R}}=0.04\right.$ and $\epsilon=0.12, \lambda_{\mathrm{R}}=0.08$ respectively, Houghton et al. 2013. $\epsilon$ is the ellipticity and $\lambda_{\mathrm{R}}$ is the specific angular momentum; slow rotators are defined as having $\lambda_{\mathrm{R}}<0.31 \sqrt{\epsilon}$, see Emsellem et al. 2011); as well as the BCG NGC4839 within the Coma south-west cluster. We also obtain unresolved spectroscopy for the fast rotator NGC4873 $\left(\epsilon=0.23, \lambda_{\mathrm{R}}=0.41\right)$ as a low- $\sigma_{*}$ comparison galaxy. Our sample covers a wide range of central velocity dispersions and metallicities (e.g. Trager et al. 2008; Loubser et al. 2009). Each of these properties have been purported as possible mechanisms for IMF variations (e.g. $[\alpha / \mathrm{Fe}]$ : Conroy \& van Dokkum 2012b; $\sigma_{*}$ : Conroy \& van Dokkum 2012b; Ferreras et al. 2013; La Barbera et al. 2013; Spiniello et al. 2014; [Z/H]: Martín-Navarro et al. 2015a), so our sample covers a suitable range to probe these correlations, and in particular a wide range of $\sigma_{*}$, which has been the most widely reported correlation. The IMF of Coma galaxies has been previously studied by Smith et al. (2012) using spectra stacked by velocity dispersion. However, their results only concerned the central $0.6 \mathrm{kpc}$. In this study for each BCG we present radial measurements of the far red features covered by the SWIFT instrument out to $10 \operatorname{arcsec}(5 \mathrm{kpc})$. We then utilise the latest stellar population synthesis (SPS) models to analyse our results in the context of a possible variable IMF.

This paper is organised as follows: Section 2 details our observations and data reduction procedures. In Section 3 we present the absorption feature strengths, and Section 4 contains our analysis using index-index diagrams and comparing to SPS model predictions for SSP spectra. We discuss our results in Section 5 and conclude our work in Section 6. In this paper we adopt the IMF naming convention used by Conroy \& van Dokkum (2012a, hereafter CvD12), namely a Chabrier IMF corresponding to that defined in Chabrier (2003) for the disc of the Milky Way; a Salpeter (1955) IMF defined as $x=2.35$; and a bottom-light IMF as defined in van Dokkum (2008). We also refer to the Kroupa (2001) and Chabrier (2003) IMFs, which are very similar in form, interchangeably as 'Milky-Way' IMFs. We adopt a flat $\Lambda \mathrm{CDM}$ cosmology with $H_{0}=68 \mathrm{~km} \mathrm{~s}^{-1} \mathrm{Mpc}^{-1}, \Omega_{m}=0.3$ and $\Omega_{\Lambda}=0.7$. This gives a distance to the Coma cluster $(z=0.024$, Han \& Mould 1992) of $108 \mathrm{Mpc}$ and an angular scale of $0.5 \operatorname{arcsec} \mathrm{kpc}^{-1}$.

\section{OBSERVATIONS AND DATA REDUCTION}

Observations were obtained over the nights of 4th May 2009; 11th May 2012; 17th, 20th and 22nd April 2013 using the SWIFT instrument (Thatte et al. 2006) on the Palomar 200 inch $(5.1 \mathrm{~m})$ telescope. Observations were taken at the 235 mas spaxel ${ }^{-1}$ spatial scale covering a field of $10^{\prime \prime} \times 20^{\prime \prime}$. The spectra cover the wavelength range $6300-10400 \AA$ with a dispersion of $\sim 2 \AA$ FWHM and a sampling of $1 \AA \mathrm{pix}^{-1}$. Table 1 lists our galaxy sample and total exposure time for each galaxy. The seeing was around $1.5^{\prime \prime}$ for all observations.

\subsection{Data reduction}

The data were reduced using the SWIFT data reduction pipeline, written in IRAF (Houghton, in preparation). The 
Table 1. Our sample of Coma galaxies with positions, effective radii $R_{\mathrm{e}}$ taken from Loubser et al. (2008) and Houghton et al. (2013), and total on-source exposure times. The $R_{\mathrm{e}}$ column shows the fraction of $R_{\mathrm{e}}$ covered by our observations in parentheses.

\begin{tabular}{lcccc}
\hline Galaxy & RA & DEC & $R_{\mathrm{e}}\left({ }^{\prime \prime}\right)$ & $T_{\exp }(\mathrm{s})$ \\
\hline NGC4889 & $13: 00: 08.1$ & $+27: 58: 37$ & $38.0(0.2)$ & 5400 \\
NGC4874 & $12: 59: 35.7$ & $+27: 57: 33$ & $50.4(0.2)$ & 6300 \\
NGC4839 & $12: 57: 24.3$ & $+27: 29: 52$ & $17.2(0.5)$ & 3600 \\
NGC4873 & $12: 59: 32.8$ & $+27: 59: 01$ & $5.9(0.5)$ & 2700 \\
\hline
\end{tabular}

pipeline handles all the standard reduction processes of bias subtraction, flat fielding, wavelength calibration, error propagation as well as IFS specific features of illumination correction and data cube reconstruction. Cosmic rays were detected and removed using the LACOSMIC routine (van Dokkum 2001). First-order sky subtraction was performed by subtracting sky data cubes observed adjacent in time to each science cube. We were careful to choose sky fields as to avoid contamination from other galaxies in the cluster.

Residual sky lines and telluric absorption are two effects that significantly hamper accurate measurement of the faint far red absorption features of interest, especially the FeH index. The next two sub-sections detail our efforts to minimise these effects and ensure high quality spectra with which to make robust index measurements.

\subsection{Telluric correction}

Due to the redshift of the Coma cluster $(z=0.024$; Han $\&$ Mould 1992) the IMF-sensitive absorption features are shifted relative to the regions affected by telluric absorption. Fig. 1 shows an example telluric absorption spectrum (from the ESO Skycalc tool; Noll et al. 2012) convolved to the SWIFT spectral resolution and scaled by a factor of 0.5 . Also plotted is an example model spectrum (red: CvD12 13.5 Gyr Chabrier IMF SSP) redshifted to $z=0.024$, as well as an example telluric standard A0V spectrum (blue: after division by a polynomial fit to the continuum) showing the positions of the prominent Paschen absorption lines. This illustrates the regions affected by both atmospheric absorption and telluric star features, relative to the positions of the key far red absorption features in the science spectra (shaded regions). Atmospheric telluric absorption is prominent in the blue pseudo-continuum of $\mathrm{NaI}$ and across the $\mathrm{MgI}$ and $\mathrm{TiO}$ features. The furthest red pseudo-continuum region of $\mathrm{CaT}$ also falls into a telluric region, but $\mathrm{FeH}$ is in a region of negligible telluric absorption. However the SWIFT throughput is a strong function of wavelength at the red end, which must be corrected for. Furthermore, residuals from correcting the prominent Paschen feature are present around $1.01 \mu \mathrm{m}$, which can affect our measurements of the $\mathrm{FeH}$ feature. Fig. 2 shows an example telluric spectrum along with a fit to the continuum. Also shown are the FeH bandpass definitions shifted to the redshift of NGC4873 $(z=0.0193$, Trager et al. 2008). This clearly shows that our FeH measurements would be susceptible to residuals from poor fitting of the Paschen lines. We therefore pursue two separate methods to correct for telluric absorption.

Firstly, we use A0V stars observed alongside the galaxies to act as telluric standards. We remove the prominent
Paschen absorption lines using a dedicated routine written in IDL. The telluric spectra are divided out by model A0V spectra provided by R. Kurucz ${ }^{1}$, using a version of the amoeba algorithm allowing for velocity shifts and stellar rotation. Fig. B1 shows examples of our telluric correction around the MgI feature for NGC4889 ( best case), NGC4874 and NGC4839 ( worst case). In each plot we use our kinematic template fits (see section 2.3) as an assumed 'telluric free' galaxy spectrum. We compute the rms residuals after telluric correction around each feature $\left(\mathrm{NaI}_{\mathrm{SDSS}}, \mathrm{CaT}, \mathrm{MgI}\right.$, $\mathrm{TiO}$ ) for each galaxy. We find that $\mathrm{MgI}$ has the highest residuals of $\sigma \sim 0.01$ (see Fig. B1) and all other features have residuals lower than 1 per cent.

Secondly, we fit a high order polynomial to the continuum of each telluric star spectrum (after division by the best fitting A0V model) to represent a relative throughput curve without residual Paschen absorption. We then divide the galaxy by this fit to correct for the smooth throughput variation at the red end. Thus we create two separate spectra for each galaxy, one corrected by the telluric spectrum and one corrected by a continuum fit to the (A0V divided) telluric spectrum. We use the telluric corrected spectrum to measure the $\mathrm{NaI}_{\mathrm{SDSS}}$, $\mathrm{CaT}, \mathrm{MgI}$ and $\mathrm{TiO}$ features, and we use the 'throughput-divided' spectrum to measure the $\mathrm{FeH}$ feature. We compare $\mathrm{FeH}$ measurements from both methods in Fig. B3 in appendix B.

\subsection{Second-order sky subtraction, binning and kinematics}

Residual sky emission is still prominent at red wavelengths (after the first-order sky subtraction) due to time-dependent variations of the sky emission on the scale of minutes. These are the dominant source of systematic error around $1 \mu \mathrm{m}$ and so must be accurately removed to measure the FeH feature of only a few per cent strength relative to the continuum. We employ two independent methods to try and remove these residual lines. The first method involves fitting and removing the skylines while fitting the kinematics, as presented in Zieleniewski et al. (2015) for M31 and M32. The second method involves fitting each wavelength channel (narrowband image) of the data cube with a Sérsic function to separate the galaxy light from sky light.

\subsubsection{Simultaneous kinematics and sky subtraction}

For the first method we perform a simultaneous second-order sky subtraction while extracting the kinematics (Weijmans et al. 2009). We fit the kinematics of the spectra with the penalised pixel fitting routine (PPXF, Cappellari \& Emsellem 2004) using, as template spectra, the stellar population models from CvD12. We compute the kinematics in annular bins of increasing radii from the centres of each galaxy to achieve a target $\mathrm{S} / \mathrm{N}>100 \mathrm{pixel}^{-1}$ in the central part of the spectra (around $\mathrm{CaT}, \mathrm{MgI}$ and $\mathrm{TiO}$ features). Sky subtraction was accomplished using a variation of the techniques described in Davies (2007): rather than scaling a single sky spectrum covering multiple $\mathrm{OH}$ vibrational transitions, we divide the spectrum into separate regions covering each $\mathrm{OH}$ vibrational

1 http://kurucz.harvard.edu/stars/astar/ 


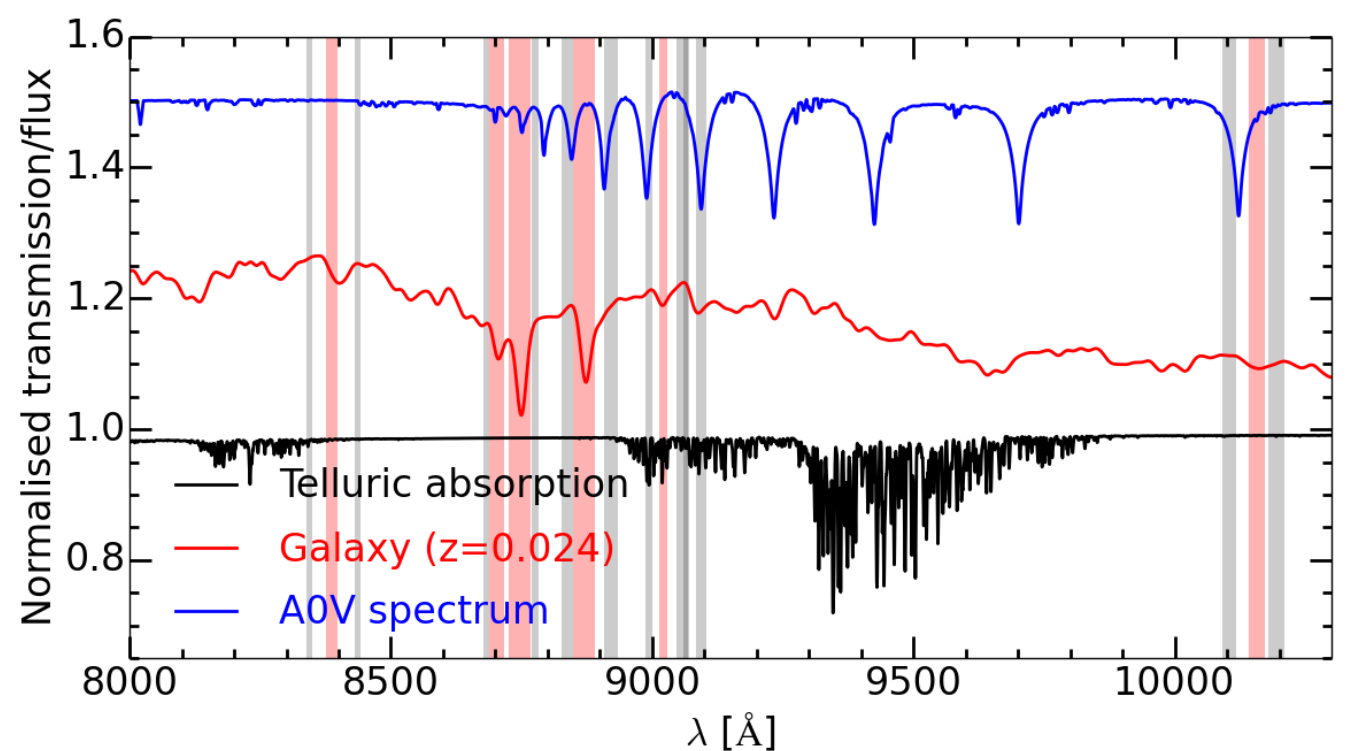

Figure 1. Plot showing atmospheric absorption spectrum (black; scaled by 0.5 and plotted at 0.98), SSP model spectrum (red; plotted at 1.2) redshifted to the nominal redshift of the Coma cluster $z=0.024$ (Han \& Mould 1992), and a model A0V spectrum (blue; continuum divided and plotted at 1.5). The telluric spectrum is convolved to the SWIFT resolution and the 'galaxy' spectrum is a CvD12 13.5 Gyr, Chabrier IMF SSP that has been smoothed to $\sigma=300 \mathrm{~km} \mathrm{~s}^{-1}$. The redshifted (to $z=0.024$ ) positions of the key absorption feature bandpasses are denoted by the shaded regions. They are, from left to right, NaI $\mathrm{SDSS}_{\text {, }}$ CaT, MgI, TiO and FeH.

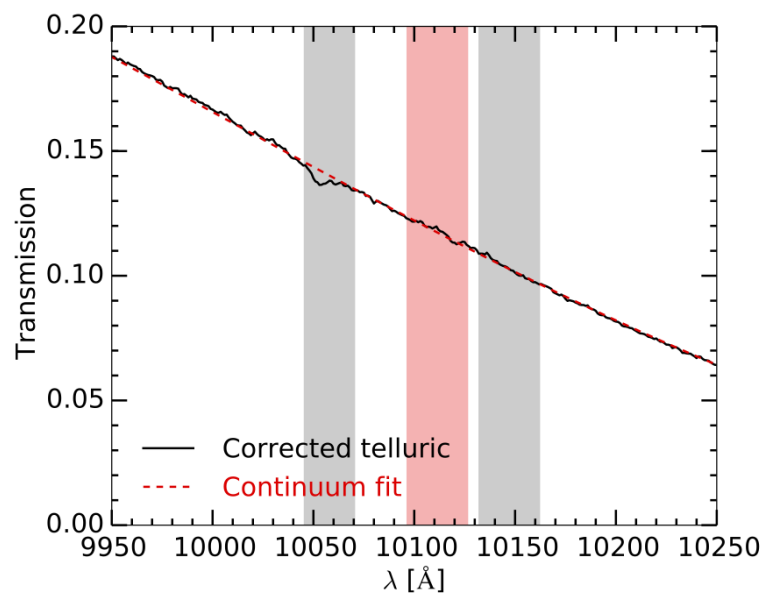

Figure 2. Plot showing the throughput, derived from a telluric spectrum, around the $\mathrm{FeH}$ feature after removing A0V features (black solid line) and a polynomial fit to the continuum of the telluric (red dashed line). A residual Paschen absorption feature is clearly evident at $10050 \AA$ which is left over from dividing out the A0V absorption. The shaded regions show the $\mathrm{FeH}$ pseudocontinuum and feature bandpass definitions redshifted to $z=$ 0.0193 of NGC4873 (Trager et al. 2008). The blue continuum would be affected by residuals in the telluric spectrum, but these are overcome by using the continuum fit. The gradient in the curve represents the varying throughput of SWIFT at the red end.

transition. This allows independent scaling of each transition. We also scale the $\mathrm{O}_{2}$ emission around $0.864 \mu \mathrm{m}$ separately. The sky spectra are extracted from a separate sky cube, which has been shifted and combined in the same way to match the spaxel positions, and from the identical annular apertures as the science spectra. All these spectra are then passed to PPXF, which finds the best-fit linear combination (including scaling) that reproduces the skyline residuals while also finding the best fit kinematics ${ }^{2}$. Furthermore, to account for flexure (error in the wavelength calibration) we shift each sky spectrum forward and backwards by three pixels when performing the fitting. For each galaxy spectrum, we measure the intrinsic instrument dispersion from the skylines at the same wavelength as the principal galaxy absorption features $(\mathrm{NaI} / \mathrm{CaT} / \mathrm{FeH})$ and independently for each azimuthal bin.

The resolution of a galaxy spectrum as observed by SWIFT is $\sigma_{*, \text { obs }}$ and is given by

$\sigma_{*, \mathrm{obs}}=\sqrt{\sigma_{*}^{2}+\sigma_{\mathrm{inst}}^{2}}$

where $\sigma_{*}$ is the intrinsic galaxy stellar velocity dispersion and $\sigma_{\text {inst }}$ is the instrument resolution. PPXF measures the galaxy resolution by fitting with template spectra of intrinsic resolution $\sigma_{\text {temp }}$. When the template resolution used in PPXF is finer than the instrument resolution $\left(\sigma_{\text {temp }}<\sigma_{\text {inst }}\right)$, we convolve the template library to the same resolution as the instrument, so $\sigma_{\text {temp,n }}=\sigma_{\text {inst }}$, and then PPXF measures,

$\sigma_{\mathrm{ppxf}} \approx \sigma_{*}=\sqrt{\sigma_{*, \mathrm{obs}}^{2}-\sigma_{\mathrm{temp}, \mathrm{n}}^{2}}$.

However, the SWIFT spectral resolution varies from $40 \mathrm{~km} \mathrm{~s}^{-1}(\mathrm{R} \sim 3100)$ to $65 \mathrm{~km} \mathrm{~s}^{-1}(\mathrm{R} \sim 2000)$ across the field of view and the resolution of the CvD12 models is $\mathrm{R}=2000$ beyond $0.75 \mu \mathrm{m}$. In this case we cannot convolve

2 We manually altered the internal limits in PPXF to allow negative sky spectrum weights. 
the template library to the instrument resolution and PPXF instead underestimates the galaxy dispersion by measuring,

$\sigma_{\mathrm{ppxf}}^{\prime}=\sqrt{\sigma_{*, \mathrm{obs}}^{2}-\sigma_{\mathrm{temp}}^{2}}$.

Therefore, when $\sigma_{\text {temp }}>\sigma_{\text {inst }}$, it is necessary to correct the dispersions found by PPXF for the difference between instrument and template resolutions. In such cases, we added in quadrature the difference between the template resolution at that wavelength range and the instrument resolution,

$\sigma_{*} \approx \sqrt{\sigma_{\text {ppxf }}^{\prime 2}+\left(\sigma_{\text {temp }}^{2}-\sigma_{\text {inst }}^{2}\right)}$.

However, in practice this only significantly affects dispersions less than $100 \mathrm{~km} \mathrm{~s}^{-1}$ so has little effect with the high dispersion galaxies presented in this study.

Using this technique, we are able to clean sky residuals from the galaxy spectra (prior to calculating element abundances) by subtracting the best-fit 'second-order' sky spectrum generated by PPXF. However, the sky spectrum contains both continuum and line emission, and scaling to remove the line residuals also scales the continuum. We check whether this can be a source of bias by adding and subtracting a constant continuum level from the galaxy spectrum and checking subsequent equivalent width measurements. We use the CvD12 Chabrier and $x=3$ IMF models and measure the indices after convolving to $400 \mathrm{~km} \mathrm{~s}^{-1}$ and adding or subtracting a constant level of 1,5 and 10 per cent. Trivially and as expected we find that the index value changes by $\sim 1,5$ and 10 per cent respectively for both IMFs. We also find that adding a first-order gradient across a feature has minor affect, on the order of 1 per cent for a 20 per cent gradient across the feature. However, we do find that a higher-order differential change in the continuum across a feature can alter the measured index more substantially; we create a high-order polynomial fit to a 10 per cent step function offset from the central feature wavelength, and adding this changes the measured indices by around 30 per cent. As there is no reason to believe continuum and line emission scale in the same way (unless the continuum is composed primarily of unresolved faint $\mathrm{OH}$ lines from the same transition or scattered light from bright OH lines Davies 2007), we subtracted the continuum from the second-order sky spectrum before using it to remove the skyline residuals in the galaxy spectra. Typically, the effect of this in equivalent width measurements is only a few per cent, but it can be as large as 10 per cent. We use a $10^{\text {th }}$ order Legendre polynomial fit with sigma-clipping to smoothly follow the continuum and minimise bias from the sky lines. We show a comparison between our first- and second-order sky subtractions in Fig. B1, and the second-order subtraction routine is shown in more detail in Fig. B2.

For each galaxy we also obtain an optimally extracted 'global' spectrum extracted over all radii. We use a method of optimal extraction based on Horne (1986) and Robertson (1986) to achieve maximal S/N. We analyse the optimally extracted global spectra (hereafter referred to as global spectra) along with the resolved data.

\subsubsection{Galaxy profile fitting}

Due to the difficulty of removing residual skylines we pursue a separate, independent sky subtraction method to compare with the PPXF method. In each galaxy object-minus-sky (O$\mathrm{S})$ data cube the galaxy light and sky light contribute to the total light differently; sky light $I_{S}$ corresponds to an additive shift up or down (assuming uniform sky across data cube), whereas galaxy light can be modelled as a Sérsic function,

$I(R)=I_{0} e^{-k R^{\frac{1}{n}}}$,

where $I$ is the intensity, $R$ is the radius, and $n$ is the Sérsic index (Sérsic 1963), and an increase in galaxy light causes a steepening of the light profile towards the centre. Thus by fitting a Sérsic profile to each wavelength channel (spatial image) of the data cube we can separate the two contributions of galaxy and sky. This profile fitting relies on the galaxy light not being uniform across the SWIFT field of view - the steeper the variation in galaxy light the better our ability to disentangle sky and galaxy. We perform this procedure for each O-S data cube. We iterate over all wavelengths and first perform a 'free' fit of,

$I(R)+I_{S}$,

using the amoeba algorithm and allowing all parameters to vary. Then we fit a 'fixed' Sérsic to each wavelength channel, varying only $I_{0}$ and $I_{S}$ with the other variables fixed at the mean values from the 'free' fit. Finally we perform another fixed fit after masking out any bad IFU slices where the fixed fit varies from the science data cube by over two standard deviations.

We are left with sky-subtracted O-S data cubes, which we combine to create an alternative sky-subtracted data cube for each galaxy. We compute the binning and kinematics using PPXF as before but without performing secondorder sky subtraction described in Section 2.3. Thus we are left with three different spectra for each galaxy radial bin (and three different global spectra), which we summarise here:

(a) telluric corrected spectra sky-subtracted using PPXF,

(b) throughput corrected spectra sky-subtracted using PPXF,

(c) throughput corrected spectra sky-subtracted using O-S spatial fitting.

Spectra (a) are used for measuring NaI, CaT, MgI and TiO features. Spectra (b) are used for measuring the $\mathrm{FeH}$ feature and we compare to measurements from spectra (a) and (c) to see the effects of telluric correction and sky-subtraction methods on the $\mathrm{FeH}$ index.

\subsubsection{Masking}

After the telluric correction and sky-subtraction routines a small number of the resolved spectra still contain bad pixels near index features or continuum definitions. We mask these regions out and replace with values from our best fit kinematic template spectra. No masking is required for any NGC4889 or NGC4873 spectra. NGC4874 has two narrow bad pixel regions around $\mathrm{CaT}$ and a bad region in the blue continuum of MgI. The outermost spectrum of NGC4839 suffers from a large number of bad regions around $\mathrm{NaI}$ and $\mathrm{CaT}$ and we treat these index measurements with some caution. No masking is required for the global spectra. 
Table 2. Median S/N of spectra around each index for each galaxy. Global refers to the optimally extracted spectrum extracted over all radii for each galaxy.

\begin{tabular}{|c|c|c|c|c|c|}
\hline $\mathrm{R}\left({ }^{\prime \prime}\right)$ & $\mathrm{NaI}_{\text {SDSS }}$ & $\mathrm{CaT}$ & MgI & $\mathrm{TiO}$ & $\mathrm{FeH}$ \\
\hline \multicolumn{6}{|c|}{ NGC4889 } \\
\hline 0.0 & 121 & 123 & 124 & 124 & 65 \\
\hline 1.7 & 155 & 159 & 160 & 160 & 83 \\
\hline 2.6 & 156 & 160 & 163 & 164 & 82 \\
\hline 3.6 & 144 & 147 & 152 & 154 & 76 \\
\hline 4.5 & 127 & 133 & 141 & 144 & 67 \\
\hline 5.4 & 112 & 116 & 123 & 127 & 58 \\
\hline 6.3 & 91 & 95 & 101 & 105 & 48 \\
\hline 7.5 & 90 & 92 & 99 & 102 & 48 \\
\hline Global & 349 & 355 & 368 & 372 & 200 \\
\hline \multicolumn{6}{|c|}{ NGC4874 } \\
\hline 0.0 & 66 & 67 & 70 & 70 & 33 \\
\hline 1.8 & 96 & 99 & 103 & 104 & 49 \\
\hline 2.8 & 94 & 96 & 102 & 104 & 48 \\
\hline 3.7 & 93 & 96 & 102 & 104 & 47 \\
\hline 4.8 & 90 & 94 & 100 & 103 & 45 \\
\hline 6.8 & 100 & 105 & 114 & 118 & 51 \\
\hline Global & 213 & 215 & 226 & 231 & 115 \\
\hline \multicolumn{6}{|c|}{ NGC4839 } \\
\hline 0.0 & 63 & 64 & 66 & 67 & 35 \\
\hline 2.0 & 99 & 101 & 107 & 108 & 55 \\
\hline 5.7 & 67 & 67 & 75 & 76 & 44 \\
\hline Global & 142 & 145 & 154 & 155 & 85 \\
\hline \multicolumn{6}{|c|}{ NGC4873 } \\
\hline Global & 96 & 95 & 105 & 105 & 54 \\
\hline
\end{tabular}

Table 3. Optical and far red index bandpass and continuum definitions used in this paper. The optical definitions are taken from Worthey et al. (1994), and the far red definitions are taken from Cenarro et al. (2001) and Conroy \& van Dokkum (2012a). In this work we use the $\mathrm{NaI}_{\mathrm{SDSS}}$ index as defined in La Barbera et al. (2013). The TiO index is defined as the ratio between the blue and red pseudo-continua. Wavelengths are in vacuum.

\begin{tabular}{|c|c|c|c|}
\hline Index & $\begin{array}{c}\text { Blue Continuum } \\
(\AA)\end{array}$ & $\begin{array}{l}\text { Feature } \\
(\AA)\end{array}$ & $\begin{array}{c}\text { Red Continuum } \\
(\AA)\end{array}$ \\
\hline \multicolumn{4}{|c|}{ Optical features } \\
\hline $\mathrm{H} \beta$ & $4829.2-4849.2$ & $4849.2-4878.0$ & $4878.0-4893.0$ \\
\hline Fe52 & $5234.7-5249.7$ & $5247.2-5287.2$ & $5287.2-5319.7$ \\
\hline $\mathrm{Fe} 53$ & $5306.1-5317.4$ & $5313.6-5353.6$ & $5354.9-5364.9$ \\
\hline $\operatorname{Mg} b$ & 5144.1-5162.8 & $5161.6-5194.1$ & $5192.8-5207.8$ \\
\hline \multicolumn{4}{|c|}{ Far red features } \\
\hline $\mathrm{NaI}_{\text {SDSS }}$ & $8145.2-8155.2$ & $8182.3-8202.3$ & $8235.3-8246.3$ \\
\hline \multirow[t]{3}{*}{$\mathrm{CaT}$} & $8474.0-8484.0$ & $8484.0-8513.0$ & $8563.0-8577.0$ \\
\hline & $8474.0-8484.0$ & $8522.0-8562.0$ & $8563.0-8577.0$ \\
\hline & $8619.0-8642.0$ & $8642.0-8682.0$ & $8700.0-8725.0$ \\
\hline MgI & 8777.4-8789.4 & 8801.9-8816.9 & 8847.4-8857.4 \\
\hline $\mathrm{TiO}$ & $8835.0-8855.0$ & & $8870.0-8890.0$ \\
\hline $\mathrm{FeH}$ & $9855.0-9880.0$ & 9905.0-9935.0 & 9940.0-9970.0 \\
\hline
\end{tabular}

\subsection{Index measurements}

The $\mathrm{S} / \mathrm{N}$ of our spectra around each index are shown in Table 2. Before making index measurements, we de-redshift each spectrum to correct for its velocity as determined from the kinematic fit.

The global galaxy spectra are shown in Fig. 3. The re- solved spectra around the NaI, CaT and FeH features are plotted for each of the BCGs in Figs. 4, 5 and 6, and the for global spectra of NGC4873 in Fig. 7.

In order to equally compare the subsequent equivalent width measurements within each galaxy, as well as between galaxies, we correct all our index measurements to a common dispersion of $200 \mathrm{~km} \mathrm{~s}^{-1}$. We describe this procedure in Appendix A. We measure the index equivalent widths using the formalism for generic indices given in Cenarro et al. (2001), which includes an error-weighted least-squares fit to the pseudo-continuum. We propagate individual pixel photon errors through the data reduction pipeline as variance spectra for each science spectrum, and present our index measurements with formal $1 \sigma$ uncertainties.

\subsubsection{Index measurement robustness}

To test the robustness of our results, we derive systematic uncertainties on the $\mathrm{FeH}$ index measurements from our multiple sky subtraction methods. The spectra and measurements are presented fully in Appendix B. We use the spread of our $\mathrm{FeH}$ measurements as a systematic uncertainty on our results in the following sections.

\section{RESULTS}

\subsection{Global spectra}

For each galaxy we have a global spectrum optimally extracted over all radii. Fig. 8 shows the index measurements from these global spectra as white-filled symbols located at 4 arcsec, along with the resolved spectra at different radii (colour filled symbols). For NGC4873 we only measure indices from the global spectra. For completeness we present the global index measurements and uncertainties in Table 4, measured at a common resolution of $\sigma=200 \mathrm{~km} \mathrm{~s}^{-1}$.

\subsection{Resolved spectra}

Radial gradients of absorption are shown in Fig. 8 and we discuss the trends for each galaxy in turn. For NGC4873 our data did not have sufficient $\mathrm{S} / \mathrm{N}$ to create resolved spectra, so this section covers only the three BCGs.

\subsubsection{NGC4889}

Strong negative gradients are evident for $\mathrm{NaI}_{\mathrm{SDSS}}$, CaT and MgI. Sodium ranges from $\sim 1.0 \AA$ in the central bin $(0.5 \mathrm{kpc})$ to $\sim 0.6 \AA$ at $8 \operatorname{arcsec}(4 \mathrm{kpc})$. Calcium ranges from $7.6 \AA$ in the central bin to flat at $\sim 6.5 \AA$ from $5 \operatorname{arcsec}(2.5 \mathrm{kpc})$ outwards. Magnesium ranges from $\sim 0.7 \AA$ in the central $2 \operatorname{arcsec}(1 \mathrm{kpc})$ to $\sim 0.25 \AA$ at 8 arcsec. A weak negative gradient in $\mathrm{TiO}$ is evident, varying from 1.081 in the central $3 \operatorname{arcsec}(1.5 \mathrm{kpc})$ to 1.075 from $6 \operatorname{arcsec}(3 \mathrm{kpc})$. Finally, FeH shows a flat profile throughout of around $0.30 \AA$, although the random and systematic uncertainties place it anywhere between $0.1-0.5 \AA$. 


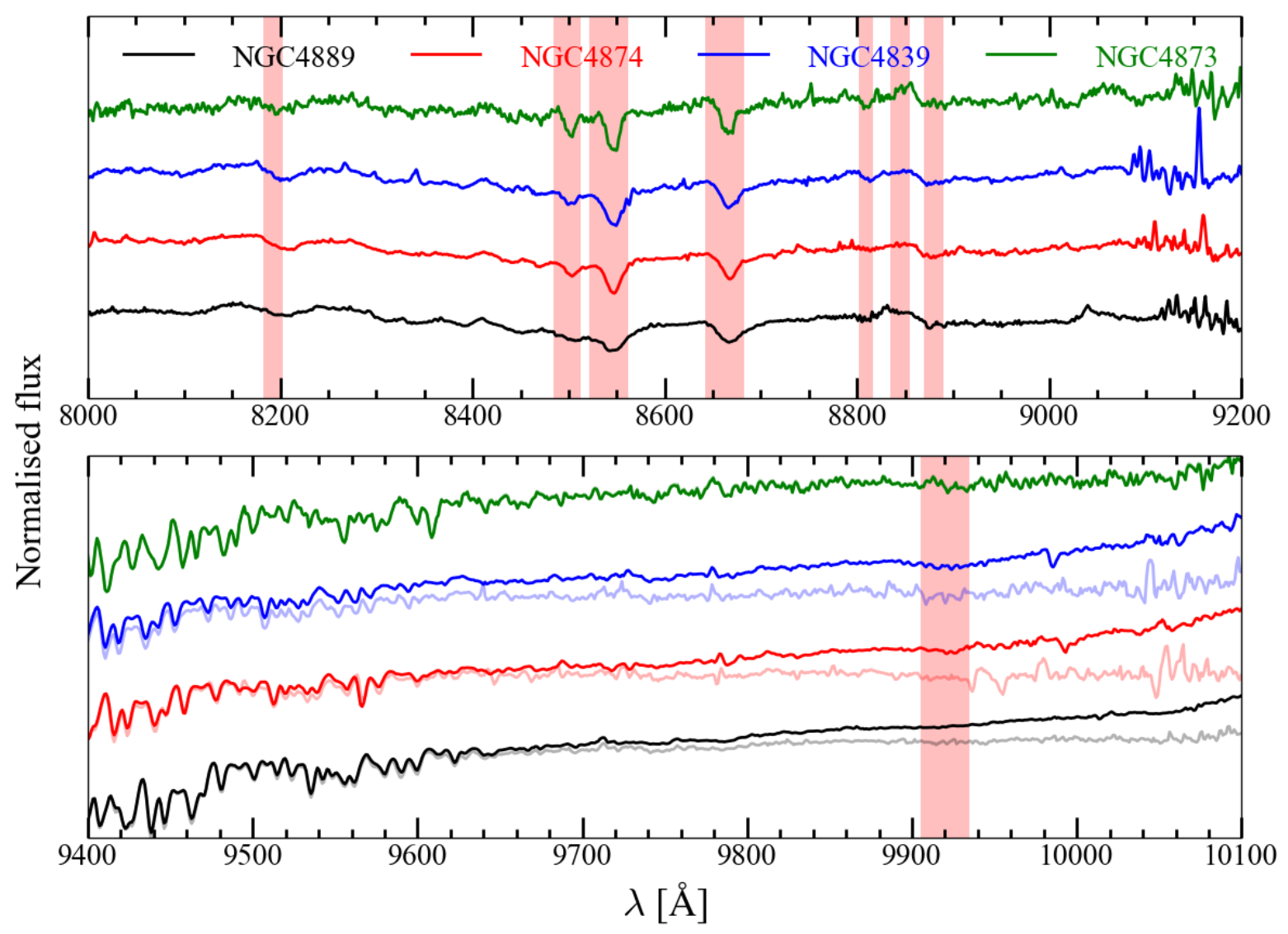

Figure 3. Global (optimally extracted) spectra of the four Coma galaxies NGC4889 (black), NGC4874 (red), NGC4839 (blue) and NGC4873 (green). Spectra have been normalised by the median continuum level and are vertically spaced for presentation purposes. In the lower panel the fainter coloured lines show the global spectra obtained using the O-S cube Sérsic profile fitting discussed in section 2.3.2. The spectra in the lower panel have also been divided by a continuum fit to the telluric spectra and so still show telluric features around $9100-9600 \AA$. The red shaded regions indicate the bandpass positions for the five far red indices.
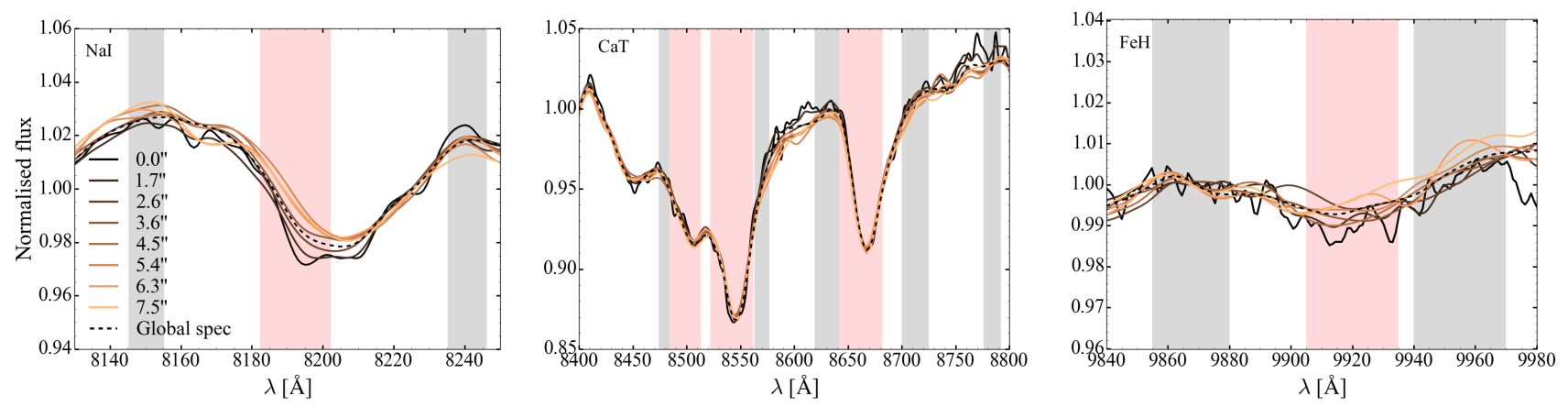

Figure 4. Spectra showing the three IMF-sensitive features in NGC4889: from left to right: $\mathrm{NaI}_{\text {sdss }}$, CaT and FeH. The spectra have been convolved to a common velocity dispersion of $400 \mathrm{~km} \mathrm{~s}^{-1}$. The spectra are coloured by radial distance in arcsec from the galaxy centre. Also plotted is the optimally extracted global spectrum (black dashed line). The limits of the feature and pseudo-continua band definitions are shown as the shaded red and grey regions respectively.

\subsection{2 $N G C 4874$}

Negative gradients are evident in $\mathrm{CaT}$ and $\mathrm{MgI}$, and flat profiles for NaISDSS, $\mathrm{TiO}$ and $\mathrm{FeH}$. Calcium ranges from $7.1 \AA$ in the central bin to $6.3 \AA$ at 8 arcsec. Magnesium ranges from $\sim 0.6 \AA$ in the central $\sim 3 \operatorname{arcsec}$ to $0.4 \AA$ at 8 arcsec. Sodium absorption is flat at $\sim 0.6 \AA$ throughout the central 6 arcsec with the outermost bin showing weaker absorption at $0.3 \AA$, although we see from Fig. 5 that the red continuum of the outermost spectrum has been strongly affected by residual sky over-subtraction. TiO shows similar behaviour with a flat profile at 1.060 to 5 arcsec and a slight decrease to 1.055 at $\sim 7$ arcsec. FeH absorption scatters around $0.4 \AA$ at 

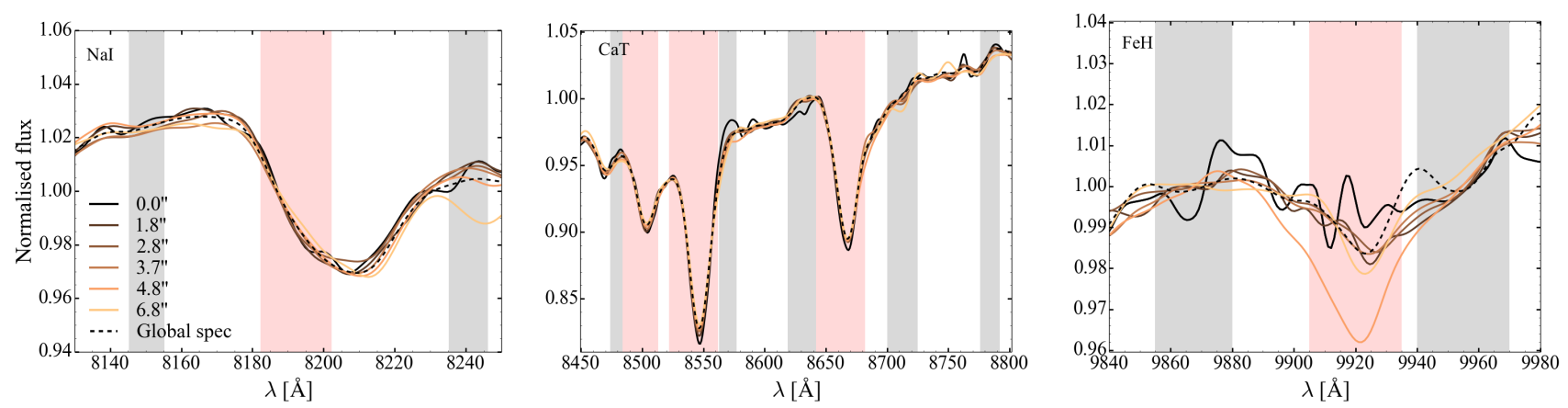

Figure 5. Same as Fig. 4 but for NGC4874: spectra showing the three IMF-sensitive features in NGC4874: from left to right: NaI sdss $_{\text {, }}$ CaT and FeH. These spectra are convolved to $270 \mathrm{~km} \mathrm{~s}^{-1}$.
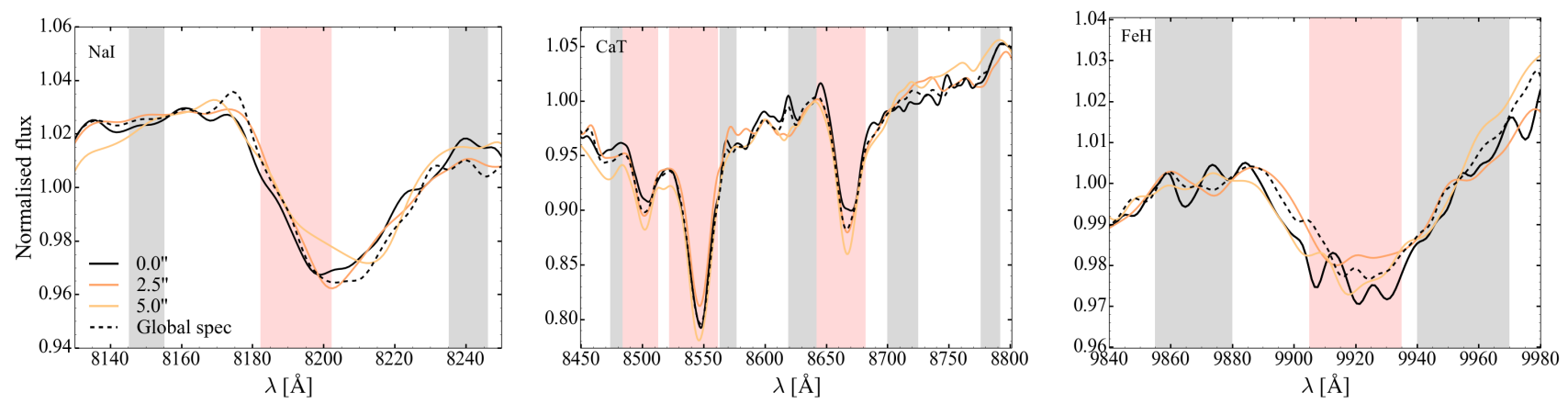

Figure 6. Same as Fig. 4 but for NGC4839: spectra showing the three IMF-sensitive features in NGC4839: from left to right: NaI sdss $_{\text {, }}$ CaT and FeH. These spectra are convolved to $270 \mathrm{~km} \mathrm{~s}^{-1}$.
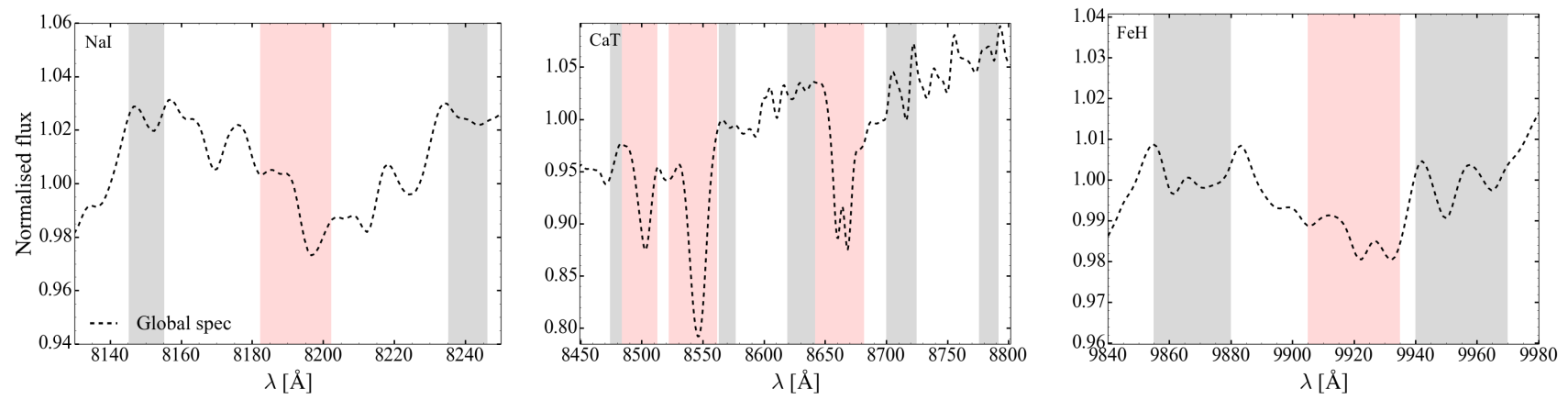

Figure 7. Same as Fig. 4 but for NGC4873: global spectra showing the three IMF-sensitive features in NGC4873: from left to right: $\mathrm{NaI}_{\text {sdss }}$, CaT and FeH. These spectra are convolved to $185 \mathrm{~km} \mathrm{~s}^{-1}$.

Table 4. Index equivalent widths from the optimally extracted global spectra for each galaxy, measured at a common velocity dispersion of $\sigma=200 \mathrm{~km} \mathrm{~s}^{-1}$. For each BCG, for the $\mathrm{FeH}$ feature we present both the random uncertainty and $\left(\begin{array}{r}\min \\ \text { min }\end{array}\right)$ values due to systematic variation from our sky subtraction procedures.

\begin{tabular}{lccccc}
\hline Galaxy & $\begin{array}{c}\text { NaI } \\
(\AA)\end{array}$ & $\begin{array}{c}\text { CaT } \\
(\AA)\end{array}$ & $\begin{array}{c}\text { MgI } \\
(\AA)\end{array}$ & TiO & $\begin{array}{c}\text { FeH } \\
(\AA)\end{array}$ \\
\hline NGC4889 & $0.80 \pm 0.02$ & $6.87 \pm 0.05$ & $0.42 \pm 0.02$ & $1.078 \pm 0.001$ & $0.30 \pm 0.04\left(\begin{array}{c}0.30 \\
0.19\end{array}\right)$ \\
NGC4874 & $0.53 \pm 0.03$ & $6.58 \pm 0.09$ & $0.19 \pm 0.02$ & $1.057 \pm 0.001$ & $0.39 \pm 0.07\left(\begin{array}{c}0.43 \\
0.33\end{array}\right)$ \\
NGC4839 & $0.68 \pm 0.05$ & $6.92 \pm 0.13$ & $0.35 \pm 0.03$ & $1.059 \pm 0.002$ & $0.64 \pm 0.10\left(\begin{array}{c}0.64 \\
0.20\end{array}\right)$ \\
NGC4873 & $0.62 \pm 0.07$ & $7.65 \pm 0.12$ & $0.59 \pm 0.03$ & $1.071 \pm 0.002$ & $0.42 \pm 0.14$ \\
\hline
\end{tabular}


all radii but carries a large uncertainty. We also note one clear anomalous FeH measurement at 5 arcsec showing very strong absorption.

\subsubsection{NGC4839}

Due to the shorter total exposure time on this galaxy we only create three radial bins with sufficient $\mathrm{S} / \mathrm{N}$ to make measurements. The outermost spectrum is also strongly affected by residual sky features, which are marked on Fig. 8 with dashed error bar lines for $\mathrm{NaI}_{\mathrm{SDSS}}$ and $\mathrm{CaT}$. The sodium feature shows strong central absorption of $\sim 0.8 \AA$ with a negative gradient to $0.7 \AA$ at $3 \operatorname{arcsec}(1.5 \mathrm{kpc})$. Calcium displays flat absorption of $\sim 6.6 \AA$ out to 3 arcsec. MgI shows absorption at $0.45 \AA$ in the central 3 arcsec and the second and third bins suggest a positive gradient up to $\sim 0.65 \AA$ around 6 arcsec. TiO displays a flat profile at a similar level to NGC4874 of $\sim 1.060$. FeH shows a strong central feature of $\sim 0.8 \AA$, lowering to a flat profile of between $0.5-0.6 \AA$. However, the large random and systematic uncertainties on this galaxy's $\mathrm{FeH}$ measurements place the measurements anywhere between $0.2-0.7 \AA$ for all other points except the central value. Therefore there is possible suggestion that NGC4839 shows a stronger $\mathrm{FeH}$ feature compared to the two BCGs in the main Coma cluster.

\section{ANALYSIS}

In this section we compare our measurements with SPS model predictions, in combination with previously published optical indices. We firstly use optical indices to constrain the age, metallicity $[\mathrm{Z} / \mathrm{H}], \alpha$-enhancement $[\alpha / \mathrm{Fe}]$ and ironenhancement $[\mathrm{Fe} / \mathrm{H}]$ of the galaxies, before using the far red indices to constrain the IMF slope.

\subsection{Stellar population synthesis models}

We make use of two sets of SPS models. For the optical features we use the Vazdekis et al. (2015, hereafter V15)

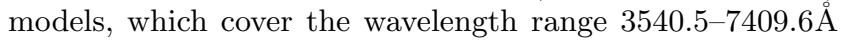
for a range of IMFs, ages, metallicities, and $\alpha$-enhancements. We use the V15 models with BaSTI isochrones (Pietrinferni et al. 2004, 2006) as only these currently provide spectra covering variations in $[\alpha / \mathrm{Fe}]$.

We compare our far-red index measurements with measurements from the SPS models of CvD12. These provide SSP spectra covering the wavelength range 1500-24000 with variations in the IMF slope ('bottom-light' through to $x=3$ 'bottom-heavy'), age (3-13.5 Gyr), $\alpha$-element enhancement $[\alpha / \mathrm{Fe}](0.0-+0.3)$, and variations in individual elemental abundance ratios $[\mathrm{X} / \mathrm{Fe}]$ for 17 different elements (for fixed 13.5 Gyr age and fixed Chabrier IMF). The 'base set' of CvD12 models (variations in age and IMF) are at solar metallicity, whereas the SSPs with variations in $[\alpha / \mathrm{Fe}]$ and $[\mathrm{X} / \mathrm{Fe}]$ are at fixed $[\mathrm{Fe} / \mathrm{H}]=0.0$, so the total metallicity $[\mathrm{Z} / \mathrm{H}]$ also changes according to the relation between metallicity, iron abundance and $\alpha$-enhancement from Trager et al. (2000),

$[\mathrm{Fe} / \mathrm{H}]=[\mathrm{Z} / \mathrm{H}]-A[\alpha / \mathrm{Fe}]$,

where $A=0.93$ (Conroy, priv. comm.). However, these are not a complete representation of enhanced metallicity SSPS as only the $\alpha$-elements are increased. When we derive IMF slopes in Section 5.1 we try to account for stellar population variations across the galaxies. We discuss the caveats of our approach and show the variations to be only second-order effects in Appendix C.

\subsection{Stellar populations from published optical measures}

The stellar populations of the three Coma BCGs have been well studied using optical spectra (e.g. Jørgensen 1999; Mehlert et al. 2000; Moore et al. 2002; Mehlert et al. 2003; Nelan et al. 2005; Sánchez-Blázquez et al. 2006; Trager et al. 2008; Loubser et al. 2009; Coccato et al. 2010; Loubser \& Sánchez-Blázquez 2012; Groenewald \& Loubser 2014). Trager et al. (2008) and Loubser et al. (2009) summarise the ages, metallicities and $\alpha$-enhancements derived through Lick indices $(\mathrm{H} \beta, \mathrm{Mg} b, \mathrm{Fe} 52, \mathrm{Fe} 53)$ for each galaxy from the literature. The values are derived using 2.7 arcsec equivalent apertures and thus represent the central regions of each galaxy.

Trager et al. (2008) and Loubser et al. (2009) have presented Lick index measurements for the centres of the galaxies in our sample, derived from apertures of 2.7 arcsec and $R_{\mathrm{e}} / 8$ in diameter respectively. In Fig. 9 we plot $[\mathrm{MgFe}]^{\prime}-$ $\mathrm{H} \beta$ (top) and $\mathrm{Mg} b-<\mathrm{Fe}>$ (bottom) index-index diagrams and compare published optical measurements for the Coma galaxies to the V15 SPS models. $<\mathrm{Fe}>$ is a combination of the Fe52 and Fe53 indices, defined as,

$<\mathrm{Fe}>=(\mathrm{Fe} 52+\mathrm{Fe} 53) / 2$,

and $[\mathrm{MgFe}]^{\prime}$ is a combination of the Fe indices with $\mathrm{Mg} b$, defined by Thomas et al. (2005) as,

$[\mathrm{MgFe}]^{\prime}=\sqrt{(\mathrm{Mg} b) \times(0.72 \times \mathrm{Fe} 52+0.28 \times \mathrm{Fe} 53)}$.

All measurements are at a common resolution of $\sigma=$ $200 \mathrm{~km} \mathrm{~s}^{-1}$, corresponding to the Lick index resolution of these features (Worthey \& Ottaviani 1997). The [MgFe]'$\mathrm{H} \beta$ diagram shows variations in age and metallicity from the V15 models as labelled on the subplot. These two indices are known to be reasonably independent of $\alpha$-enhancement and are completely independent of IMF. Comparing data to models, the galaxies are all consistently old at $\sim 13.5 \mathrm{Gyr}$. The metallicities range from $[\mathrm{Z} / \mathrm{H}] \sim+0.05$ for NGC4873, $[\mathrm{Z} / \mathrm{H}] \sim+0.25$ for NGC4839, $[\mathrm{Z} / \mathrm{H}] \sim+0.3$ for NGC4874, and $[\mathrm{Z} / \mathrm{H}] \sim+0.5$ for NGC4889.

The $\mathrm{Mg} b-<\mathrm{Fe}>$ plot shows variations in age, metallicity and $[\alpha / \mathrm{Fe}]$ from the V15 models as labelled on the subplot. All the galaxies are consistent with an $\alpha$-enhancement of $[\alpha / \mathrm{Fe}] \sim+0.25$. The metallicities are consistent with those derived from the $[\mathrm{MgFe}]^{\prime}-\mathrm{H} \beta$ plot.

\subsection{Analysis of far red features}

In Figs. 10 and 11 we plot $\mathrm{FeH}-\mathrm{TiO}$ and $\mathrm{NaI}_{\text {SDSS }}-\mathrm{MgI}$ indexindex diagrams showing 13.5 Gyr CvD12 model predictions (labelled) and our galaxy measurements (labelled in legend) at a common resolution of $200 \mathrm{~km} \mathrm{~s}^{-1}$. From the $\mathrm{FeH}-\mathrm{TiO}$ diagram we see from the CvD12 models that these indices work in orthogonal directions; $\mathrm{FeH}$ positively correlates with 


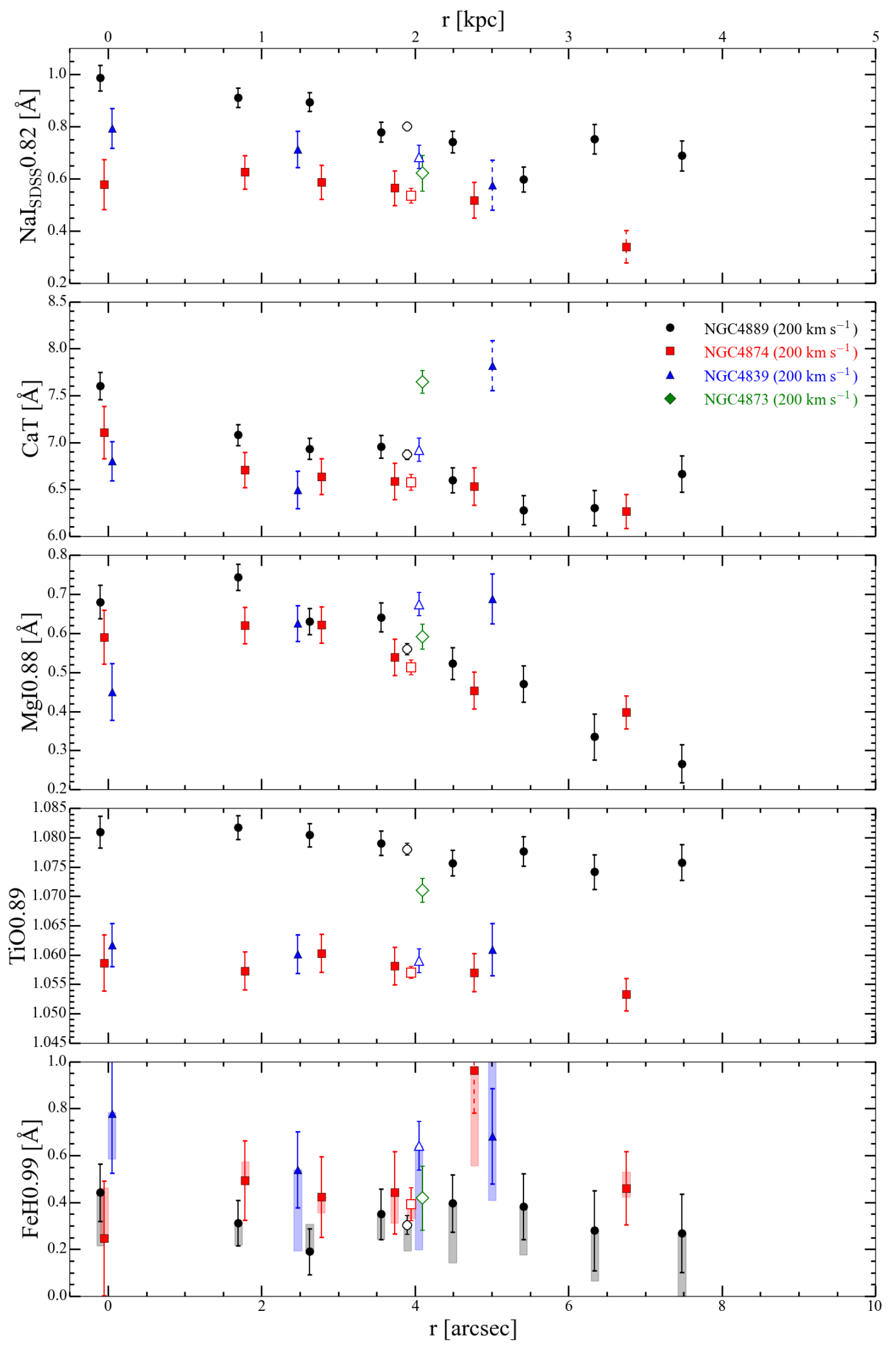

Figure 8. Plots of radial gradients for each index for NGC4889 (black circles), NGC4874 (red squares), NGC4839 (blue triangles), and NGC4873 (green diamonds). The index equivalent width measurements have been corrected to a common velocity dispersion of $200 \mathrm{~km} \mathrm{~s}^{-1}$. Plotted in white-filled symbols at $4^{\prime \prime}$ are the values from the optimally extracted global spectra. The points with dashed error bar lines have been affected by prominent residual sky. In the FeH sub-panel the shaded rectangles show the systematic uncertainty for each BGC measurement, derived through the various sky subtraction methods detailed in Sections 2.3 and 2.3.2. The individual FeH measurements from each reduction method are shown for each BCG in Fig. B3. 

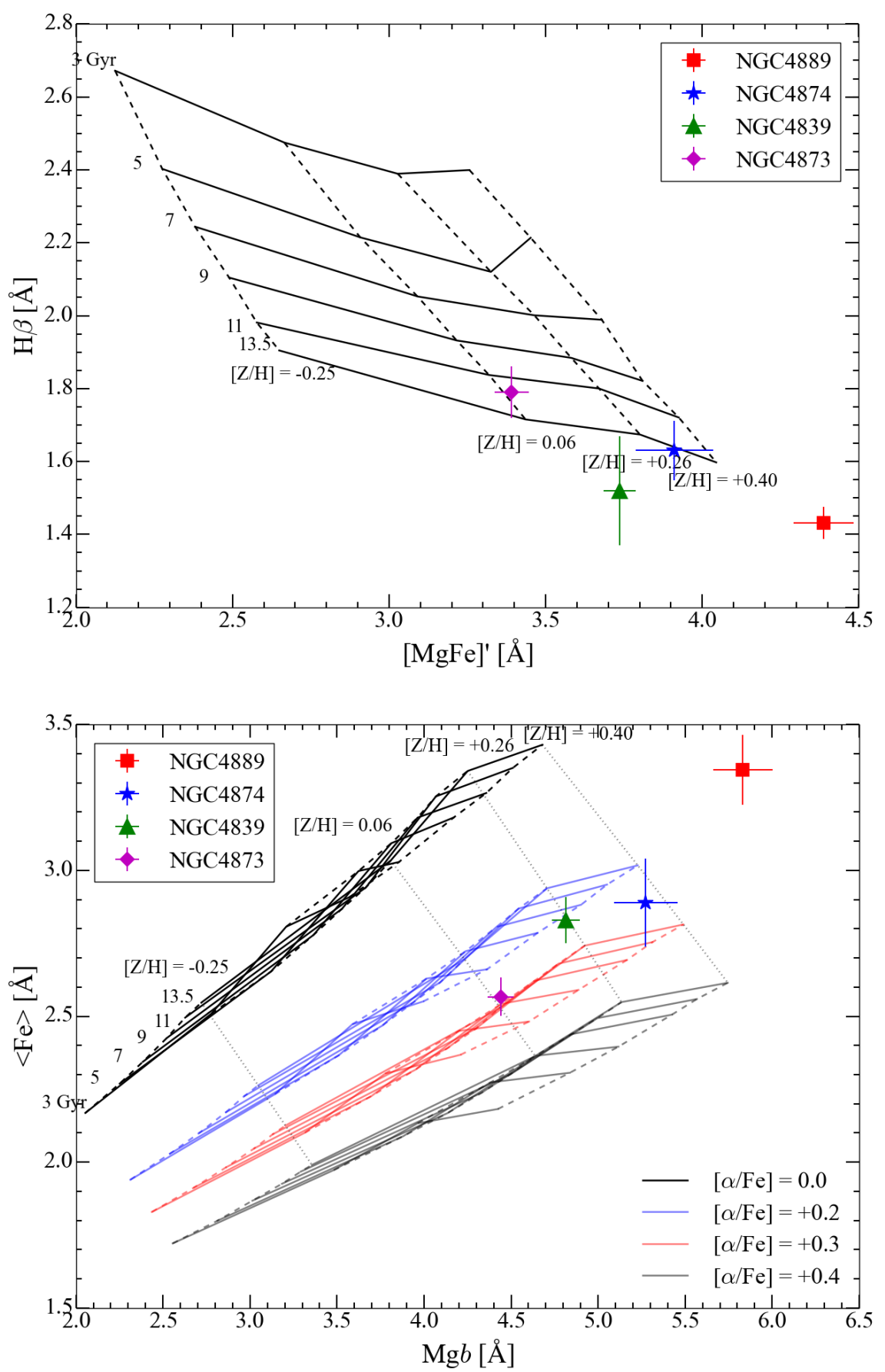

Figure 9. Top shows the $[\mathrm{MgFe}]^{\prime}-\mathrm{H} \beta$ index-index diagram showing positions of the four galaxies in our sample, compared with the SPS model predictions of Vazdekis et al. (2015, hereafter V15). The data points are taken from published results in the literature; the data points for the three BCGs represent values from Trager et al. (2008) and Loubser et al. (2009), measured from 2.7" aperture spectra and $R_{\mathrm{e}} / 8$ aperture spectra respectively. The V15 models show variation in age and total metallicity for Kroupa IMF (both indices are insensitive to IMF). $\mathrm{H} \beta$ traces variations in age and the $[\alpha / \mathrm{Fe}]$-independent $[\mathrm{MgFe}]^{\prime}$ index traces total metallicity. The data points and models are both at a common velocity dispersion of $\sigma=200 \mathrm{~km} \mathrm{~s}^{-1}$, corresponding to the Lick index resolution. All galaxies show similar ages of $\sim 13.5 \mathrm{Gyr}$, with NGC4889 showing the highest metallicity at $[\mathrm{Z} / \mathrm{H}] \sim 0.5$, NGC4873 at $[\mathrm{Z} / \mathrm{H}] \sim 0.3, \mathrm{NGC4839}$ at $[\mathrm{Z} / \mathrm{H}] \sim 0.25$ (note the weaker $\mathrm{H} \beta$ strength possibly due to emission) and $\mathrm{NGC4873}$ at $[\mathrm{Z} / \mathrm{H}] \sim 0.06$. Bottom shows the $\mathrm{Mg} b-<\mathrm{Fe}>$ index-index diagram showing positions of the four galaxies in our sample, compared with the V15 SPS model predictions for a Kroupa IMF (both indices are again insensitive to IMF). The data points are taken from published results in the literature; the data points for the three BCGs represent values from Loubser et al. (2009), and the point for NGC4873 represents the average from values in Trager et al. (2008). The data points and models are both at a common velocity dispersion of $\sigma=200 \mathrm{~km} \mathrm{~s}^{-1}$, corresponding to the Lick index resolution. All galaxies are $\alpha$-enhanced with $[\alpha / \mathrm{Fe}] \sim+0.25$. 


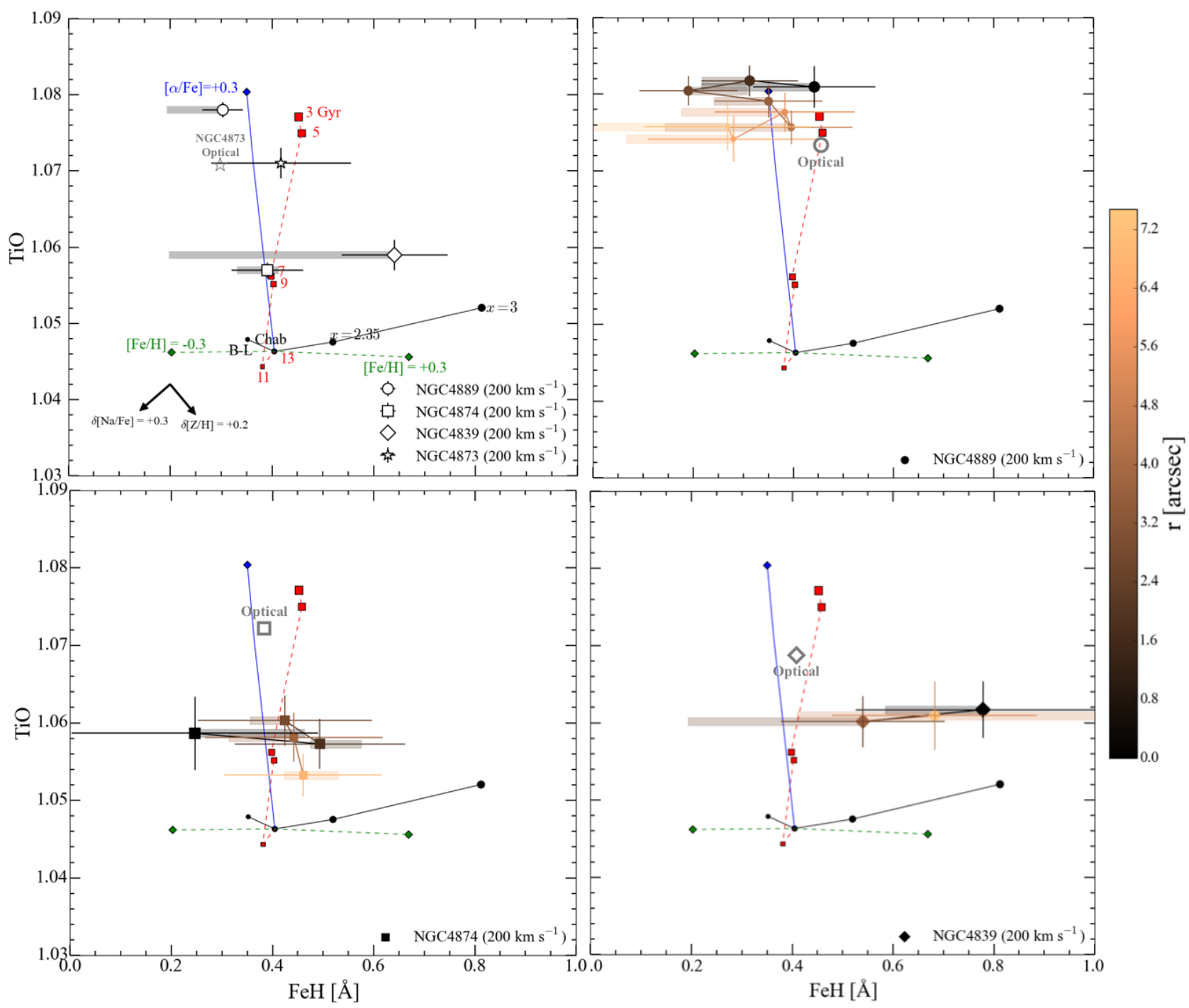

Figure 10. FeH-TiO index-index diagrams showing the global positions (top left sub-panel) and radial variation (other three subpanels) of our galaxies as shown in the legends, compared with model predictions of CvD12 at 13.5 Gyrs. In the top left plot the white filled markers show the optimally extracted global spectrum measurements for each galaxy. Data and models are compared at a common resolution of $\sigma=200 \mathrm{~km} \mathrm{~s}^{-1}$. In the bottom plot the colour bar and marker sizes indicate the radial position from the centre of each galaxy; size decreases and colour lightens with increasing radial distance. The $\mathrm{FeH}$ index measurements also show the systematic uncertainties as shaded rectangles. The CvD12 models show variation in IMF slope (black circles with each IMF labelled; $\mathrm{B}-\mathrm{L}$ for bottom-light, and Chab for Chabrier), age (red squares), $[\alpha / \mathrm{Fe}]$ (blue diamonds), and individual elemental abundance variations for a 13.5 Gyr Chabrier IMF (green diamonds). The black arrows show the index responses to changes in: total metallicity as derived from the V15 models (the FeH response is approximated from the vector shown in Fig. 10 of La Barbera et al. (2016)), and sodium abundance as derived from the CvD12 models. In each sub-panel, the grey marker labelled Optical shows the reference position for each galaxy based upon the optical indices from Fig. 9, assuming a Chabrier IMF and with no over/under abundance of individual elements. For NGC4889 (circles), we infer an old, $\alpha$-enhanced population with a Chabrier, or possibly bottom-light, IMF. For NGC4874 we infer a Chabrier IMF, whereas for NGC4839 we see some evidence of a bottom-heavy $(x>2.35)$ IMF in the centre, although with a very large systematic uncertainty. NGC4873 is also fully consistent with an Chabrier IMF.

IMF slope and $[\mathrm{Fe} / \mathrm{H}]$, whereas $\mathrm{TiO}$ increases with increasing $[\alpha / \mathrm{Fe}]$. The $\mathrm{NaI}_{\mathrm{SDSS}}-\mathrm{MgI}$ diagram shows that $\mathrm{MgI}$ is independent of IMF, positively correlates with $[\mathrm{Z} / \mathrm{H}]$ and neg-

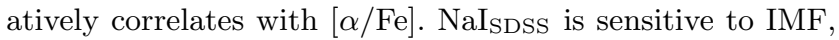
$\alpha$ - and sodium abundance. We now discuss the analysis of each galaxy in turn.

\subsection{NGC4889}

For NGC4889, from Fig. 9 and Equation 7 we adopt an age of $13.5 \mathrm{Gyr}$, metallicity of $[\mathrm{Z} / \mathrm{H}]=+0.5, \alpha$-enhancement of $[\alpha / \mathrm{Fe}]=+0.25$, and iron-enhancement of $[\mathrm{Fe} / \mathrm{H}]=+0.27$.

Fig. 10 shows the NGC4889 data points with circle markers. In the FeH-TiO subplot the NGC4889 measurements scatter around the $[\alpha / \mathrm{Fe}]=+0.3,13.5$ Gyr Chabrier IMF SSP, with no radial trends. The $\mathrm{FeH}$ measurements reject a bottom-heavy $x=3$ IMF slope and the global spec- 


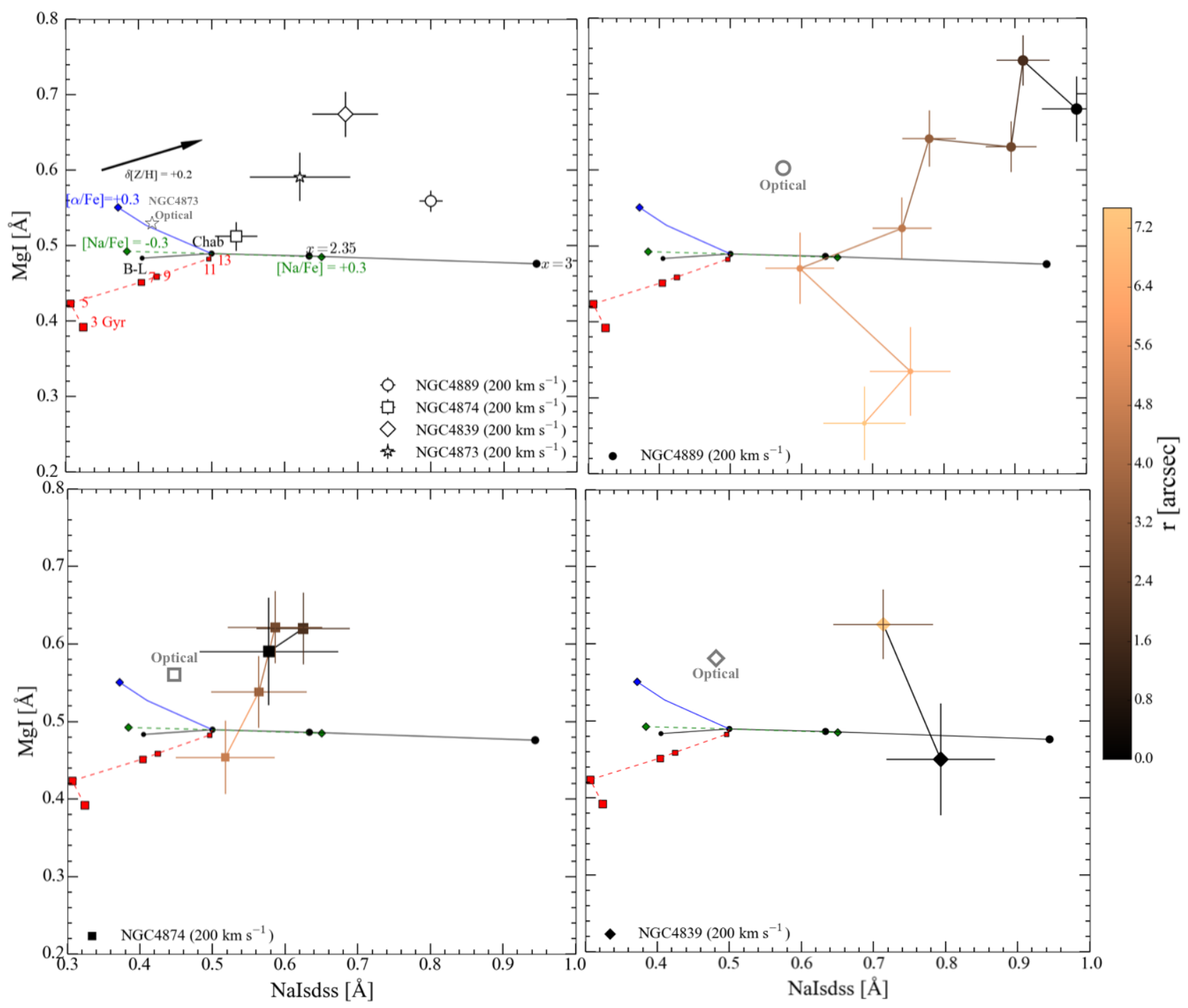

Figure 11. Same as Fig. 10 but for $\mathrm{NaI}_{\mathrm{SDSS}}-\mathrm{MgI}$ indices: diagrams showing the global positions (top left sub-panel) and radial variation (other three sub-panels) of our galaxies as shown in the legends, compared with model predictions of CvD12 at 13.5 Gyrs. In each sub-panel, the grey marker labelled Optical shows the reference position for each galaxy based upon the optical indices from Fig. 9, assuming a Chabrier IMF and with no over/under abundance of individual elements. For NGC4889 we fix the IMF as Chabrier from the $\mathrm{FeH}-\mathrm{TiO}$ diagram and infer from the $\mathrm{NaI}_{\mathrm{SDSS}}-\mathrm{MgI}$ diagram strong gradients in either the relative abundances of sodium and magnesium, or an overall metallicity gradient, rather than a bottom-heavy $x=3$ IMF. NGC4874 and NGC4873 are consistent with a Chabrier IMF and metal- and $\alpha$-enhanced populations. For NGC4839 the strong NaI IDSS measurements provide some evidence of a bottom-heavy $(x>2.35)$ IMF in the centre, although with the large $\mathrm{FeH}$ uncertainty this is also consistent with Na-enhancement.

trum measurement strongly rejects a bottom-heavy IMF. In combination with the optical indices, our measurements qualitatively suggest that NGC4889 is consistent with an old, $\alpha$-enhanced population with a Chabrier, or possibly even bottom-light, IMF.

The $\mathrm{NaI}_{\text {SDSS }}-\mathrm{MgI}$ diagram in Fig. 11 shows the radial gradients of these two indices in NGC4889. As we take the age, $[\alpha / \mathrm{Fe}]$ and IMF to be fixed from the optical indices and $\mathrm{FeH}-\mathrm{TiO}$ diagram, we infer a strong negative gradient in the sodium abundance, or a total metallicity gradient (e.g. Coccato et al. 2010), rather than the interpretation of a bottom-heavy $x=3$ IMF with decreasing age gradient.

The most striking behaviour from the radial index measurements is the discrepancy between the large NaI SDSS gradient and the flat $\mathrm{FeH}$ profile. Zieleniewski et al. (2015) and
McConnell et al. (2016) have both measured the same discrepant behaviour, in M31 and two massive ETGs respectively. These galaxies have lower central velocity dispersions between $200<\sigma_{*}<250 \mathrm{~km} \mathrm{~s}^{-1}$ and so NGC4889 represents a much higher dispersion and more massive galaxy where bottom-heavy IMFs have been recently proposed (e.g. Cappellari et al. 2012; Conroy \& van Dokkum 2012b; La Barbera et al. 2013; Ferreras et al. 2013; Spiniello et al. 2014). Furthermore, van Dokkum \& Conroy (2010) presented a stacked spectrum around $\mathrm{NaI}$ from four galaxies in the Coma cluster, which included NGC4889, and they concluded a bottomheavy IMF. However, they did not present the stacked Coma spectrum around FeH. Thus, the flat FeH profile we measure presents tension with the notion of increased IMF slope in 
this galaxy and with the general IMF- $\sigma_{*}$ relation (see Section 5.1).

A Chabrier IMF gives a sodium enhancement in the centre of $[\mathrm{Na} / \mathrm{Fe}] \sim+1.0$, decreasing to $[\mathrm{Na} / \mathrm{Fe}] \sim+0.3$ at $5 \operatorname{arcsec}(2.5 \mathrm{kpc})$. An overall metallicity trend would give $[\mathrm{Z} / \mathrm{H}] \sim+0.6$ in the centre down to $[\mathrm{Z} / \mathrm{H}] \sim+0.3$ at 10 arcsec, fully consistent with Coccato et al. (2010). We note from the $[\mathrm{Na} / \mathrm{Fe}]$ vector on Fig. 10 that Na-enhancement slightly suppresses the measured FeH strength, so the strong central Na-enhancement would act to weaken the central $\mathrm{FeH}$ measurement in NGC4889. However, this is more than offset by the $[\mathrm{Fe} / \mathrm{H}]$ vector and we have accounted for both effects when measuring the IMF slopes in Section 5.1 (see Appendix C for further details).

\subsection{NGC4874}

For NGC4874, from Fig. 9 and Equation 7 we adopt an age of $13.5 \mathrm{Gyr}$, metallicity of $[\mathrm{Z} / \mathrm{H}]=+0.25, \alpha$-enhancement of $[\alpha / \mathrm{Fe}]=+0.25$, and iron-enhancement of $[\mathrm{Fe} / \mathrm{H}]=+0.02$.

Figs. 10 and 11 show the NGC4874 data points with square markers. We have excluded the anomalously high FeH datapoint at $r=5$ arcsec and the outermost NaI SDSS $_{\text {S }}$ datapoint from these figures for clarity. The remaining $\mathrm{FeH}-$ $\mathrm{TiO}$ points scatter around the Chabrier IMF slope with a bottom-heavy $x=3 \mathrm{IMF}$ excluded. The TiO index places the galaxy at around $[\alpha / \mathrm{Fe}] \sim+0.1$ for an $13.5 \mathrm{Gyr}$ population. Similarly to NGC4889, we find from the $\mathrm{FeH}$ index that NGC4874 is consistent with a Chabrier IMF and solar iron abundance, but we cannot rule out the possibility of a dwarf-dominated IMF and iron deficiency. The $\mathrm{NaI}_{\mathrm{SDSS}^{-}}$ $\mathrm{MgI}$ diagram again suggests either a weak gradient in magnesium or an overall metallicity gradient. The flat profile of $\mathrm{NaI}_{\mathrm{SDSS}}$ at $\sim 0.4 \AA$ (excluding the outermost point which has a bad red continuum region) suggests a sodium enhancement of $[\mathrm{Na} / \mathrm{Fe}] \sim+0.2$ for a Chabrier IMF. The NaI $\mathrm{IDSS}$ and $\mathrm{FeH}$ strengths at $\sim 0.4 \AA$ strengthen the case for a Chabrier IMF in this galaxy, rather than a bottom-heavy IMF, which would require sodium deficiency to match the observed $\mathrm{NaI}_{\text {SDSS }}$ profile.

\subsection{NGC4839}

For NGC4839, from Fig. 9 and Equation 7 we adopt an age of 13.5 Gyr, metallicity of $[\mathrm{Z} / \mathrm{H}]=+0.3, \alpha$-enhancement of $[\alpha / \mathrm{Fe}]=+0.25$, and iron-enhancement of $[\mathrm{Fe} / \mathrm{H}]=0.07$.

Figs. 10 and 11 show the NGC4839 data points with diamond markers. The $\mathrm{FeH}$ measurements scatter around a bottom-heavy $x>2.35$ IMF slope and the TiO measurements place the galaxy around $[\alpha / \mathrm{Fe}] \sim+0.1$ for a 13 Gyr population. The $\mathrm{TiO}$ measurements are very similar to NGC4874. The key difference for this galaxy is the potentially deeper $\mathrm{FeH}$ absorption compared with the other two BCGs, although we note that there are large systematic uncertainties on the $\mathrm{FeH}$ measurements, and the central measurement shows a deeper strength at only the $1 \sigma$ level. The global spectrum $\mathrm{FeH}$ measurement places the galaxy at an IMF slope of $x \sim 2.5$ at the $1 \sigma$ level. From the $\mathrm{NaI}_{\mathrm{SDSS}}-\mathrm{MgI}$ diagram we infer that NGC4839 is also metal enhanced, and the strong $\mathrm{NaI}_{\text {SDSS }}$ index with no gradient also supports a possible steeper IMF slope, but is equally consistent with an Na-enhancement of $[\mathrm{Na} / \mathrm{Fe}] \sim+0.5$ for a Chabrier IMF.

\subsection{NGC4873}

For NGC4873, from Fig. 9 and Equation 7 we adopt an age of $12 \mathrm{Gyr}$, metallicity of $[\mathrm{Z} / \mathrm{H}]=+0.06, \alpha$-enhancement of $[\alpha / \mathrm{Fe}]=+0.25$, and iron-enhancement of $[\mathrm{Fe} / \mathrm{H}]=-0.17$.

Figs. 10 and 11 show the NGC4873 global data point with the star marker. Our measurements are consistent with NGC4873 being old and $\alpha$-enhanced. The FeH measurement is consistent with a Chabrier IMF from FeH. This is further supported by the $\mathrm{NaI}_{\mathrm{SDSS}}$ measurement if a sodium enhancement of $[\mathrm{Na} / \mathrm{Fe}] \sim+0.4$ is invoked.

\section{DISCUSSION}

\subsection{The IMF- $\sigma_{*}$ relation}

Ferreras et al. (2013), La Barbera et al. (2013) and Spiniello et al. (2014) have all published IMF- $\sigma_{*}$ relations for ETGs showing that an increase in $\sigma_{*}$ corresponds to an increase in IMF slope. These relations are all derived using galaxies covering a range of velocity dispersions from $\sim 150$ $320 \mathrm{~km} \mathrm{~s}^{-1}$, and so all fall well within this range. While there is some variation between the relations, the general conclusion is that galaxies with $\sigma_{*}>200 \mathrm{~km} \mathrm{~s}^{-1}$ (for LB13 and F13; $\sigma_{*}>250 \mathrm{~km} \mathrm{~s}^{-1}$ for S14) have IMFs steeper than Salpeter, and galaxies with $\sigma_{*}<200 \mathrm{~km} \mathrm{~s}^{-1}$ have IMFs approaching Chabrier. Therefore our qualitative analysis for NGC4873 $\left(\sigma_{*} \sim 200 \mathrm{~km} \mathrm{~s}^{-1}\right)$ having a normal IMF agrees well with these relations. NGC4874 and NGC4839 have $\sigma_{*} \sim 270 \mathrm{~km} \mathrm{~s}^{-1}$ and the index interpretation of NGC4839 having a steeper (than Salpeter) IMF slope also agrees. However, our global measurements for NGC4874 are not consistent with an IMF slope steeper than Salpeter. Finally, NGC4889 $\left(\sigma_{*} \sim 400 \mathrm{~km} \mathrm{~s}^{-1}\right)$ has a central dispersion much larger than the galaxies used to derive the IMF- $\sigma_{*}$ relations, but our inferred Chabrier IMF from the flat $\mathrm{FeH}$ profile is inconsistent with the relations, which suggest a very bottomheavy IMF slope $(x \sim 3)$.

To quantitatively compare our results to the published IMF- $\sigma_{*}$ relations we derive single power-law IMF slopes for our full sample of galaxies using the CvD12 models. When deriving the IMF slopes we take into account the derived values for age, $[\mathrm{Z} / \mathrm{H}],[\alpha / \mathrm{Fe}],[\mathrm{Fe} / \mathrm{H}]$ and $[\mathrm{Na} / \mathrm{Fe}]$. The method is described in Appendix C. Our derived IMF slopes for each galaxy are shown in Fig. 12 as a function of velocity dispersion. We find consistency with a Chabrier IMF for each galaxy across the $\sigma_{*}$ range, except for NGC4839 where we find possible evidence of an IMF steeper than Salpeter, although with a very large uncertainty. We also show the IMF- $\sigma_{*}$ relations derived by Ferreras et al. (2013), La Barbera et al. (2013) and Spiniello et al. (2014) using optical $\mathrm{Na}$ and $\mathrm{TiO}$ features. We discuss the caveats of our IMF derivations in Appendix C. However we note that there is negligible change in our derived values for $x$ whether we account for stellar population parameter variations between galaxies, or not.

Ferreras et al. (2013) and La Barbera et al. (2013) have also invoked a bimodal form of the IMF and fitted it to observational data to derive $\mathrm{IMF}_{\mathrm{b}}-\sigma_{*}$ relations. The bimodal IMF is defined in Vazdekis et al. (1996) with a slope of $x$ for $M>0.6 \mathrm{M}_{\odot}$ and flattening below $0.6 \mathrm{M}_{\odot}$. For an exponent of $x=2.3$ it very closely traces the Kroupa (2001) 


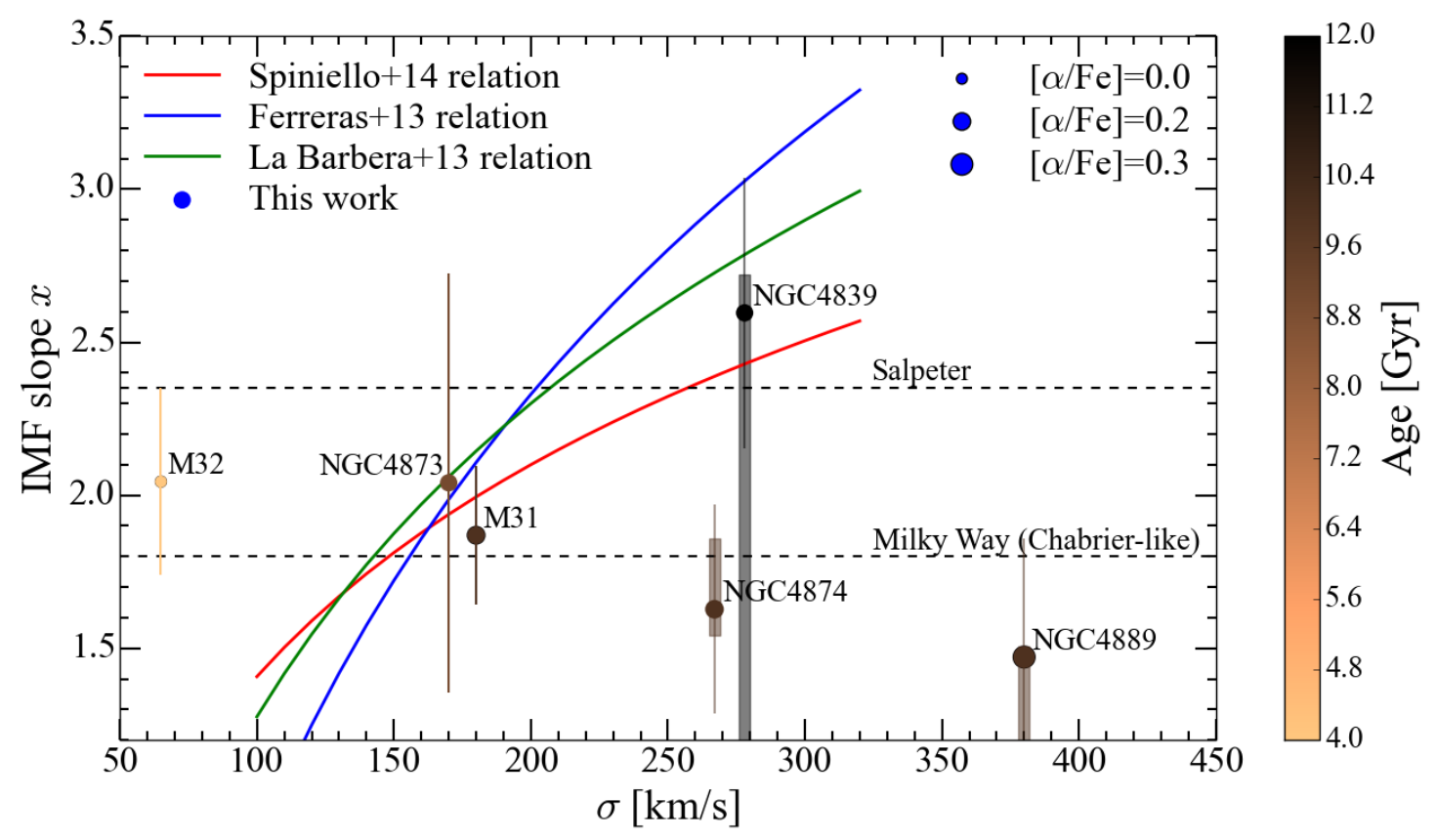

Figure 12. Derived IMF slope $x$ against central velocity dispersion for our sample of Coma galaxies, as well as M32 and M31 from Zieleniewski et al. (2015). The IMF slope has been derived from our global FeH measurements accounting for CvD12 model responses to age, $[\alpha / \mathrm{Fe}]$ and $[\mathrm{Fe} / \mathrm{H}]$ variations, as described in Section 5.1, using published values in the literature for each galaxy (Rose et al. 2005; Trager et al. 2008; Loubser et al. 2009; Saglia et al. 2010; Coccato et al. 2010; Loubser \& Sánchez-Blázquez 2012). For our data, the colour of each point indicates the age and the marker size indicates the level of $[\alpha / \mathrm{Fe}]$ enhancement for each galaxy. The coloured lines show unimodal IMF- $\sigma_{*}$ relations from the previous works of Ferreras et al. (2013); La Barbera et al. (2013) and Spiniello et al. (2014), derived using central 2.7" SDSS spectra and several optical/NIR features, including NaD/NaI but excluding FeH. The dashed horizontal lines show the Salpeter and Milky-Way (Chabrier-like) slopes. Our results show consistency with a universal Chabrier IMF across a large range of velocity dispersions, with the exception of the single galaxy NGC4839, for which we see possible evidence of an IMF slightly heavier than Salpeter, albeit with a very large systematic uncertainty.

universal IMF. La Barbera et al. (2016) recently used the $\mathrm{FeH}$ index to distinguish between bottom-heavy unimodal and bimodal IMFs in a massive ETG $\left(\sigma_{*} \sim 300 \mathrm{~km} \mathrm{~s}^{-1}\right)$. FeH is most sensitive to $M \leqslant 0.3 M_{\odot}$ and thus can constrain the very low mass end of the IMF. From La Barbera et al. (2016) the FeH index is predicted to vary much more weakly with increasing $x$, compared to the unimodal IMF. In fact a bottom-heavy bimodal IMF with $x=4$ predicts a similar FeH strength to the Salpeter IMF (at $\sigma_{*}=300 \mathrm{~km} \mathrm{~s}^{-1}$; see Figure 10 in La Barbera et al. 2016) of $\sim 0.45 \AA$. However, NGC4889 has an even weaker $\mathrm{FeH}$ feature at a larger $\sigma_{*}$ and seems to be incompatible with the specific, extreme bimodal bottom-heavy IMF slope of $x=4$ (for $M>0.6 \mathrm{M}_{\odot}$ ) proposed in La Barbera et al. (2016).

\subsection{Comparison with dynamical modelling and lensing results}

Stellar $M_{*} / L_{R}$ have been published for each of the BCGs by Thomas et al. $(2007,2011)$ and for NGC4889 by McConnell et al. (2012). For NGC4889, the result by Thomas et al. (2007) of $M_{*} / L_{R}=6.5 \mathrm{M}_{\odot} \mathrm{L}_{\odot}{ }^{-1}$ (Thomas et al. do not quote $1 \sigma$ uncertainties) and by McConnell et al. (2012) of $M_{*} / L_{R}=5.9 \pm 1.7 \mathrm{M}_{\odot} \mathrm{L}_{\odot}{ }^{-1}$ are in good agreement. For NGC4874 and NGC4839, Thomas et al. (2007, 2011) measured $M_{*} / L_{R}=7.0 \mathrm{M}_{\odot} \mathrm{L}_{\odot}{ }^{-1}$ and $M_{*} / L_{R}=$
$8.5 \pm 2.0 \mathrm{M}_{\odot} \mathrm{L}_{\odot}{ }^{-1}$ respectively. It should be noted that these represent upper limits on $M_{*} / L$ due to the uncertainties of incorporating a dark matter component in the models (e.g. Thomas et al. 2011; Bundy et al. 2015).

The order of increasing $M_{*} / L_{R}$ between NGC4889, NGC4874 and NGC4839 is echoed by the IMFs we infer from the $\mathrm{FeH}$ measurements within these galaxies. Calculating $M / L_{R}$ from the best-fit SSPs used to derive the IMF slope for each galaxy, for NGC4889 we measure $M_{*} / L_{R}=$ $7.2 \pm 2.0 \mathrm{M}_{\odot} \mathrm{L}_{\odot}{ }^{-1}$, for NGC4874 we measure $M_{*} / L_{R}=$ $5.5 \pm 1.0 \mathrm{M}_{\odot} \mathrm{L}_{\odot}{ }^{-1}$, and for NGC4839 we measure $M_{*} / L_{R}=$ $8.7 \pm 1.5 \mathrm{M}_{\odot} \mathrm{L}_{\odot}{ }^{-1}$. These mass-to-light ratios from SSP models are in general agreement with the published dynamical measurements.

Mass-to-light ratios measured from gravitationally lensed galaxies also provide an interesting comparison. Smith \& Lucey (2013) have presented a massive lensed ETG $\left(\sigma_{*} \approx 330 \mathrm{~km} \mathrm{~s}^{-1}\right)$ with a mass excess factor $\alpha=$ $(M / L)_{\text {lens }} /(M / L)_{\text {kroup }}=1.04 \pm 0.15$, which strongly favours a Kroupa or Chabrier IMF and rejects even a Salpeter IMF. Furthermore, Smith et al. (2015a) have presented an average mass excess factor derived from three gravitationally lensed massive ETGs $\left(\sigma_{*}>300 \mathrm{~km} \mathrm{~s}^{-1}\right)$. They find $\langle\alpha\rangle=1.1$, which again rejects a Salpeter IMF and strongly rejects bottom-heavy IMFs in these galaxies. Thus, our results are 
consistent with IMF determinations for other massive ETGs using independent methods.

\subsection{IMF or abundance gradients and implications for the stellar populations of BCGs}

Strong radial gradients of absorption features have now been documented at small radii in some ETGs by several authors, most notably for sodium (e.g. Faber \& French 1980; Boroson \& Thompson 1991; Martín-Navarro et al. 2015a,b; Zieleniewski et al. 2015; McConnell et al. 2016). Furthermore, the discrepancy between the strengths of sodium and iron 'IMF' indices has also been noted by numerous authors (e.g. Smith et al. 2012; Conroy \& van Dokkum 2012a; Smith \& Lucey 2013; Zieleniewski et al. 2015; McConnell et al. 2016). This poses interesting questions for understanding the physical context of these gradients. The general scenario is that BCGs form in the deepest potential wells in short, intense star formation bursts, with successive growth through small-scale mergers around the outskirts (e.g. Larson 1974; Carlberg 1984; Naab et al. 2009; Hopkins et al. 2010; Cappellari 2016). This inside-out formation scenario predicts metallicity gradients, as the central stellar populations enrich in-falling gas sustaining the ongoing star formation. For NGC4889 we infer a total metallicity, or magnesium and sodium abundance, gradients. Tracing the radial behaviour of the NaD feature would help further constrain the underlying physical driver behind the $\mathrm{NaI}_{\text {SDSS }}$ gradient. However, sodium is not an $\alpha$ element and is generally poorly understood in the context of galaxy stellar populations (e.g. Jeong et al. 2013; Spiniello et al. 2014; Smith et al. 2015b), so further study of sodiumenhancement in galaxies is required.

The recent study of IMF variations has shown correlations with both $[\alpha / \mathrm{Fe}]$ and central velocity dispersion (Cappellari et al. 2012; Conroy \& van Dokkum 2012b; Ferreras et al. 2013; La Barbera et al. 2013; Spiniello et al. 2014). One physical model is that larger mass and $\alpha$-enhanced systems have more type II supernovae driven turbulence, leading to greater fragmentation of star forming gas clouds (Conroy \& van Dokkum 2012b). Chabrier et al. (2014) highlights that the IMF slope should correlate with density rather than velocity dispersion. Therefore, galaxies that underwent more rapid formation periods via mergers or intense gas inflows should have more bottom-heavy IMFs. NGC4839 sits in a sub-cluster located to the south west of the main Coma cluster. Our FeH measurements for this galaxy hint at the possibility of a different formation density compared to the other two BCGs, and so this would make an exciting object for follow-up observations targeting different IMF-sensitive features.

The very low FeH measured in NGC4889 could be consistent with a bottom-light IMF dominated by remnants rather than low-mass stars. This form has been proposed by studies of sub-millimetre galaxies with extremely high star formation rates, which have been thought to be the progenitors of BCGs (e.g. Baugh et al. 2005; Narayanan \& Davé 2012, 2013, c.f. Hayward et al. 2013). Indeed, the dynamical modelling of Cappellari et al. (2012, 2013a) shows a systematic increase of the $M_{*} / L$ with velocity dispersion, but cannot distinguish between bottom-light or bottom-heavy forms, so this scenario would still be consistent.

Our work, and other studies using independent meth- ods (e.g. gravitational lensing, Smith \& Lucey 2013; Smith et al. 2015a), points to the notion that careful mass determinations of individual galaxies should be taken on a galaxy-by-galaxy basis. High $\mathrm{S} / \mathrm{N}$ optical spectroscopy of these objects covering other known IMF-sensitive features (see e.g. Spiniello et al. 2014) would help elucidate the stellar component of these galaxies. Our results show that the Coma BCGs present an exciting target for IFS surveys like MaNGA (Bundy et al. 2015), which would be able to provide mass constraints using both, visible to far red spectral coverage, and dynamical modelling techniques.

\section{CONCLUSIONS}

Using the Oxford SWIFT instrument we have undertaken a study of the three brightest cluster galaxies in the Coma core, and south-west extension, with the aim of measuring radial gradients in IMF-sensitive far red stellar absorption features. We obtained high $\mathrm{S} / \mathrm{N}$ integral field spectroscopy of NGC4889, NGC4874 and NGC4839, covering the central $\sim 10 \operatorname{arcsec}(5 \mathrm{kpc})$ of each galaxy, as well as unresolved data for the fast rotator NGC4873. We developed two separate sky subtraction methods to minimise sky line residuals in our spectra and validate our results. We investigated the far red $\mathrm{NaI}$ doublet, calcium triplet $\mathrm{CaT}$, magnesium MgI, titanium oxide $\mathrm{TiO}$ and iron hydride Wing-Ford band $\mathrm{FeH}$ as functions of radius and conclude:

1. NGC4889 shows strong negative gradients in NaI $\mathrm{IDSS}_{\mathrm{S}}$ and $\mathrm{CaT}$, mild negative gradients in $\mathrm{MgI}$ and $\mathrm{TiO}$, and a flat profile of weak $\mathrm{FeH}$ absorption. The flat $\mathrm{FeH}$ profile contrasts with the strong NaI SDSS gradient in the context of spatial IMF variations. Comparing $\mathrm{FeH}$ and $\mathrm{TiO}$ indices to stellar population models we infer a Chabrier or possibly bottom-light IMF and old, $\alpha$-enhanced population, in agreement with optical stellar population studies. This result is especially interesting due to the large velocity dispersion of $\sim 400 \mathrm{~km} \mathrm{~s}^{-1}$ in the centre of NGC4889, which from previous IMF- $\sigma_{*}$ relations would infer a very bottom-heavy IMF.

2. NGC4874 shows weak negative gradients in CaT, MgI and $\mathrm{TiO}$. NaI $\mathrm{I}_{\text {SDSS }}$ and $\mathrm{FeH}$ profiles are both flat and do not show strong absorption. Comparing to models we again find a Chabrier IMF with lower $\alpha$-enhancement compared to NGC4889 and in agreement with optical stellar population studies.

3. NGC4873 displays strong $\mathrm{CaT}, \mathrm{MgI}$ and $\mathrm{TiO}$ absorption. Our measurements of $\mathrm{FeH}$ and $\mathrm{NaI}_{\text {SDSS }}$ suggest a Chabrier IMF, which is what we expect for a galaxy of this velocity dispersion.

4. Within NGC4839 we measure strong NaI and strong FeH absorption, although we note a large systematic uncertainty on $\mathrm{FeH}$ due to the sky subtraction process. There is possible evidence of a more dwarf-heavy population in the centre, consistent with Salpeter, but the large uncertainty prevents a strong conclusion. We compare to dynamical modelling results, which suggest an IMF up to $\sim 1.5-2.5$ times heavier than Kroupa is consistent, which would be in agreement with a Salpeter IMF.

5 . These galaxies span a large range of velocity dispersions and we find tension with the notion of a simple IMF$\sigma_{*}$ relation compared to the interpretation from the IMFsensitive features. Specifically, NGC4889 has a very high 
central dispersion $\left(\sim 400 \mathrm{~km} \mathrm{~s}^{-1}\right)$ and we only detect low levels of $\mathrm{FeH}$ compared with a strong NaI gradient. We conclude that interpretations of dwarf-dominated stellar populations should be treated on a galaxy-by-galaxy basis. Combining near-infrared and optical IMF-sensitive spectral indices with dynamical models should give strong constraints on the dwarf-abundance in individual galaxies.

\section{ACKNOWLEDGMENTS}

We are grateful to the anonymous referee for providing feedback that greatly improved this manuscript. The Oxford SWIFT integral field spectrograph was supported by a Marie Curie Excellence Grant from the European Commission (MEXT-CT-2003-002792, Team Leader: N. Thatte). It was also supported by additional funds from the University of Oxford Physics Department and the John Fell OUP Research Fund. Additional funds to host and support SWIFT at the 200-inch Hale Telescope on Palomar were provided by Caltech Optical Observatories.

This work was supported by the Astrophysics at Oxford grants (ST/H002456/1 and ST/K00106X/1) as well as visitors grant (ST/H504862/1) from the UK Science and Technology Facilities Council. SZ is supported by STFCHARMONI grant ST/J002216/1. RCWH was supported by the Science and Technology Facilities Council [STFC grant numbers ST/H002456/1, ST/K00106X/1 \& ST/J002216/1]. RLD acknowledges travel and computer grants from Christ Church, Oxford, and support from the Oxford Centre for Astrophysical Surveys, which is funded through generous support from the Hintze Family Charitable Foundation.

\section{REFERENCES}

Baugh C. M., Lacey C. G., Frenk C. S., Granato G. L., Silva L., Bressan A., Benson A. J., Cole S., 2005, MNRAS, 356, 1191

Boroson T. A., Thompson I. B., 1991, AJ, 101, 111

Bundy K., et al., 2015, ApJ, 798, 7

Cappellari M., 2016, preprint, (arXiv:1602.04267)

Cappellari M., Emsellem E., 2004, PASP, 116, 138

Cappellari M., et al., 2012, Nature, 484, 485

Cappellari M., et al., 2013a, MNRAS, 432, 1709

Cappellari M., et al., 2013b, MNRAS, 432, 1862

Carlberg R. G., 1984, ApJ, 286, 403

Carter D., Visvanathan N., Pickles A. J., 1986, ApJ, 311, 637

Cenarro A. J., Cardiel N., Gorgas J., Peletier R. F., Vazdekis A., Prada F., 2001, MNRAS, 326, 959

Cenarro A. J., Gorgas J., Vazdekis A., Cardiel N., Peletier R. F., 2003, MNRAS, 339, L12

Chabrier G., 2003, PASP, 115, 763

Chabrier G., Hennebelle P., Charlot S., 2014, ApJ, 796, 75

Coccato L., Gerhard O., Arnaboldi M., 2010, MNRAS, 407, L26

Conroy C., 2013, ARA\&A, 51, 393

Conroy C., van Dokkum P., 2012a, ApJ, 747, 69

Conroy C., van Dokkum P. G., 2012b, ApJ, 760, 71

Cushing M. C., Rayner J. T., Davis S. P., Vacca W. D., 2003, ApJ, 582, 1066

Davies R. I., 2007, MNRAS, 375, 1099

Emsellem E., et al., 2011, MNRAS, 414, 888

Faber S. M., French H. B., 1980, ApJ, 235, 405

Ferreras I., La Barbera F., de la Rosa I. G., Vazdekis A., de Carvalho R. R., Falcón-Barroso J., Ricciardelli E., 2013, MNRAS, 429, L15
Greene J. E., Janish R., Ma C.-P., McConnell N. J., Blakeslee J. P., Thomas J., Murphy J. D., 2015, ApJ, 807, 11

Groenewald D. N., Loubser S. I., 2014, MNRAS, 444, 808

Han M., Mould J. R., 1992, ApJ, 396, 453

Hayward C. C., Narayanan D., Kereš D., Jonsson P., Hopkins P. F., Cox T. J., Hernquist L., 2013, MNRAS, 428, 2529

Hopkins P. F., Bundy K., Hernquist L., Wuyts S., Cox T. J., 2010, MNRAS, 401, 1099

Horne K., 1986, PASP, 98, 609

Houghton R. C. W., et al., 2013, MNRAS, 436, 19

Jeong H., Yi S. K., Kyeong J., Sarzi M., Sung E.-C., Oh K., 2013, ApJS, 208, 7

Jørgensen I., 1999, MNRAS, 306, 607

Kroupa P., 2001, MNRAS, 322, 231

Kuntschner H., et al., 2010, MNRAS, 408, 97

La Barbera F., Ferreras I., Vazdekis A., de la Rosa I. G., de Carvalho R. R., Trevisan M., Falcón-Barroso J., Ricciardelli E., 2013, MNRAS, 433, 3017

La Barbera F., Vazdekis A., Ferreras I., Pasquali A., Cappellari M., Martín-Navarro I., Schönebeck F., Falcón-Barroso J., 2016, MNRAS, 457, 1468

Larson R. B., 1974, MNRAS, 166, 585

Loubser S. I., Sánchez-Blázquez P., 2012, MNRAS, 425, 841

Loubser S. I., Sansom A. E., Sánchez-Blázquez P., Soechting I. K., Bromage G. E., 2008, MNRAS, 391, 1009

Loubser S. I., Sánchez-Blázquez P., Sansom A. E., Soechting I. K., 2009, MNRAS, 398, 133

Martín-Navarro I., Barbera F. L., Vazdekis A., Falcón-Barroso J., Ferreras I., 2015a, MNRAS, 447, 1033

Martín-Navarro I., La Barbera F., Vazdekis A., Ferré-Mateu A., Trujillo I., Beasley M. A., 2015b, MNRAS, 451, 1081

McConnell N. J., Ma C.-P., Murphy J. D., Gebhardt K., Lauer T. R., Graham J. R., Wright S. A., Richstone D. O., 2012, ApJ, 756, 179

McConnell N. J., Lu J. R., Mann A. W., 2016, ApJ, 821, 39

Mehlert D., Saglia R. P., Bender R., Wegner G., 2000, A\&AS, 141,449

Mehlert D., Thomas D., Saglia R. P., Bender R., Wegner G., 2003, A\&A, 407, 423

Miller G. E., Scalo J. M., 1979, ApJS, 41, 513

Moore S. A. W., Lucey J. R., Kuntschner H., Colless M., 2002, MNRAS, 336, 382

Naab T., Johansson P. H., Ostriker J. P., 2009, ApJ, 699, L178

Narayanan D., Davé R., 2012, MNRAS, 423, 3601

Narayanan D., Davé R., 2013, MNRAS, 436, 2892

Nelan J. E., Smith R. J., Hudson M. J., Wegner G. A., Lucey J. R., Moore S. A. W., Quinney S. J., Suntzeff N. B., 2005, ApJ, 632, 137

Noll S., Kausch W., Barden M., Jones A. M., Szyszka C., Kimeswenger S., Vinther J., 2012, A\&A, 543, A92

Oliva-Altamirano P., Brough S., Jimmy Kim-Vy T., Couch W. J., McDermid R. M., Lidman C., von der Linden A., Sharp R., 2015, MNRAS, 449, 3347

Pietrinferni A., Cassisi S., Salaris M., Castelli F., 2004, ApJ, 612, 168

Pietrinferni A., Cassisi S., Salaris M., Castelli F., 2006, ApJ, 642, 797

Robertson J. G., 1986, PASP, 98, 1220

Rose J. A., Arimoto N., Caldwell N., Schiavon R. P., Vazdekis A., Yamada Y., 2005, AJ, 129, 712

Saglia R. P., et al., 2010, A\&A, 509, A61

Salpeter E. E., 1955, ApJ, 121, 161

Sánchez-Blázquez P., Gorgas J., Cardiel N., González J. J., 2006, A\&A, 457, 809

Schiavon R. P., Barbuy B., Rossi S. C. F., Milone A., 1997a, ApJ, 479, 902

Schiavon R. P., Barbuy B., Singh P. D., 1997b, ApJ, 484, 499 
Sérsic J. L., 1963, Boletin de la Asociacion Argentina de Astronomia La Plata Argentina, 6, 41

Smith R. J., Lucey J. R., 2013, MNRAS, 434, 1964

Smith R. J., Lucey J. R., Carter D., 2012, MNRAS, 426, 2994

Smith R. J., Lucey J. R., Conroy C., 2015a, MNRAS, 449, 3441

Smith R. J., Alton P., Lucey J. R., Conroy C., Carter D., 2015b, MNRAS, 454, L71

Spiniello C., Trager S. C., Koopmans L. V. E., Chen Y. P., 2012, ApJ, 753, L32

Spiniello C., Trager S., Koopmans L. V. E., Conroy C., 2014, MNRAS, 438, 1483

Thatte N., Tecza M., Clarke F., Goodsall T., Lynn J., Freeman D., Davies R. L., 2006, in Society of Photo-Optical Instrumentation Engineers (SPIE) Conference Series. p. 3, doi:10.1117/12.670859

Thomas D., Maraston C., Bender R., Mendes de Oliveira C., 2005, ApJ, 621, 673

Thomas J., Saglia R. P., Bender R., Thomas D., Gebhardt K., Magorrian J., Corsini E. M., Wegner G., 2007, MNRAS, 382, 657

Thomas J., et al., 2011, MNRAS, 415, 545

Trager S. C., Faber S. M., Worthey G., González J. J., 2000, AJ, 120,165

Trager S. C., Faber S. M., Dressler A., 2008, MNRAS, 386, 715

Treu T., Auger M. W., Koopmans L. V. E., Gavazzi R., Marshall P. J., Bolton A. S., 2010, ApJ, 709, 1195

Vazdekis A., Casuso E., Peletier R. F., Beckman J. E., 1996, ApJS, 106, 307

Vazdekis A., Ricciardelli E., Cenarro A. J., Rivero-González J. G., Díaz-García L. A., Falcón-Barroso J., 2012, MNRAS, 424, 157

Vazdekis A., et al., 2015, MNRAS, 449, 1177

Weijmans A.-M., et al., 2009, MNRAS, 398, 561

Wing R. F., Ford Jr. W. K., 1969, PASP, 81, 527

Worthey G., Ottaviani D. L., 1997, ApJS, 111, 377

Worthey G., Faber S. M., Gonzalez J. J., Burstein D., 1994, ApJS, 94,687

Zieleniewski S., Houghton R. C. W., Thatte N., Davies R. L., 2015, MNRAS, 452, 597

van Dokkum P. G., 2001, PASP, 113, 1420

van Dokkum P. G., 2008, ApJ, 674, 29

van Dokkum P. G., Conroy C., 2010, Nature, 468, 940

van Dokkum P. G., Conroy C., 2012, ApJ, 760, 70

\section{APPENDIX A: CORRECTING TO A COMMON VELOCITY DISPERSION}

In order to equally compare our equivalent width measurements within each galaxy, as well as between galaxies, we apply the following procedure to correct for varying velocity dispersions. We first make index measurements $I\left(\sigma_{*}\right)$ at the intrinsic velocity dispersions of each spectrum. For each feature we use the CvD12 models to derive a correction factor, which is applied to the index measurement to scale it to a specific velocity dispersion. For each model SSP spectrum, we convolve it up to a range of $\sigma$ from $80-410 \mathrm{~km} \mathrm{~s}^{-1}$ and measure the index values at each $\sigma$. These $I(\sigma)$ curves are then normalised to be unity at the velocity dispersion of the galaxy spectrum $\sigma_{*}$, so we then take the average value of the correction factor,

$C(\sigma)=I\left(\sigma_{*}\right) / I(\sigma)$,

at the velocity dispersion we are correcting to and divide each galaxy index measurement by its corresponding factor. We use the CvD12 SPS models to derive the correction factors $C(\sigma)$ for our index measurements. Fig. A1 shows $C(\sigma)$ as a function of velocity dispersion $\sigma$ for the $\mathrm{FeH}$ index from a subset of CvD12 SSP spectra as shown in the legend. The correction factor is normalised to be unity at the intrinsic velocity dispersion of the galaxy spectrum and then the galaxy intrinsic index measurement is divided by the average value at the 'correcting' dispersion, for which in this paper we use $\sigma=200 \mathrm{~km} \mathrm{~s}^{-1}$. Fig. A1 shows the curves normalised at a value of $\sigma=270 \mathrm{~km} \mathrm{~s}^{-1}$, which then gives an value of $C\left(\sigma_{200}\right)=0.93$.

The CvD12 SSPs cover a range of stellar population parameters that appropriate for the galaxies studied in this paper. These include Chabrier and $x=3$ bottom-heavy IMFs, 9 and 13.5 Gyr ages, and solar and $\alpha$-enhanced (and therefore metal-enhanced) abundances. The spread of the curves introduces an additional uncertainty when correcting the index measurements to a different $\sigma$. Table A1 shows the maximum additional uncertainty for each galaxy when correcting its central spectrum's indices, which have the largest velocity dispersions within each galaxy, to $\sigma=200 \mathrm{~km} \mathrm{~s}^{-1}$.

\section{APPENDIX B: TELLURIC CORRECTION, SKY SUBTRACTION, AND WING-FORD MEASUREMENTS}

We show here additional plots concerning the accuracy of our telluric correction and sky subtraction techniques. Fig. B1 shows the telluric correction around MgI (left-hand side) and the PPXF sky subtraction around FeH (right-hand side) for each of the BCGs; NGC4889 (top row), NGC4874 (middle row) and NGC4839 (bottom row). For each galaxy, the $\mathrm{MgI}$ region is the worst affected by telluric absorption and we show the residuals for this region. We find smaller residuals around the $\mathrm{NaI}_{\text {SDSS }}$, $\mathrm{CaT}$ and $\mathrm{TiO}$ features of no worse than 0.01 . We do not incur residuals around the $\mathrm{FeH}$ feature as we divide by a smooth fit to the throughput curve instead of by a corrected telluric spectrum. The right hand plots show the first- and second-order sky subtractions on central spectra around $\mathrm{FeH}$ using PPXF. Black lines show the original spectrum with no sky subtraction. Blue lines show the spectrum after first-order O-S cube sky subtraction; the first-order subtraction is achieved using a static sky data cube of the same size as the science cube (with separate cubes correctly shifted as to match spaxel positions). Green lines show the final spectrum after second-order PPXF sky subtraction; the second-order correction is performed using PPXF and removes the residuals at the level of a few percent left over from the first-order subtraction.

Fig. B2 shows the global and radial spectra around $\mathrm{FeH}$ for each BCG, before and after the two second-order sky subtraction routines: the PPXF method on the left hand side, and the Sérsic profile fitting on the right hand side. The top two panels show NGC4889, the middle two show NGC4874 and the bottom two show NGC4839. On the left hand panels, the input spectra to PPXF (red lines) are compared to the corrected spectra in black and blue. The sky regions that are scaled by PPXF to fit the sky lines in the science spectra are shown by the vertical lines. The black line output spectra are the results when scaling all sky regions shown by the red and blue vertical lines. The blue output spectra are the results when removing the sky regions shown by the vertical blue dashed-dotted lines and only scaling a sin- 
Table A1. Maximum additional error and $(\underset{\min }{\max })$ of $C\left(\sigma_{200}\right)$ from correcting to a common velocity dispersion of $\sigma=200 \mathrm{~km} \mathrm{~s}^{-1}$. The error is calculated as the standard deviation of all values of $C\left(\sigma_{200}\right)$ from each SSP as shown in Fig. A1, when correcting from the maximal (central) velocity dispersion of each galaxy.

\begin{tabular}{|c|c|c|c|c|c|}
\hline Galaxy & $\sigma_{\mathrm{NaI}_{\mathrm{SDSS}}}$ & $\sigma_{\mathrm{CaT}}$ & $\sigma_{\mathrm{MgI}}$ & $\sigma_{\mathrm{TiO}}$ & $\sigma_{\mathrm{FeH}}$ \\
\hline G & $0.016\left(\begin{array}{l}0.826 \\
0.779\end{array}\right)$ & $0.005\left(\begin{array}{l}0.818 \\
0.805\end{array}\right)$ & $0.035\left(\begin{array}{l}0.432 \\
0.322\end{array}\right)$ & $0.003\left(\begin{array}{l}0.988 \\
0.980\end{array}\right)$ & $0.017\left(\begin{array}{l}0.819 \\
0.776\end{array}\right)$ \\
\hline NGC4874 & $0.007\left(\begin{array}{l}0.943 \\
0.922\end{array}\right)$ & $0.002\left(\begin{array}{l}0.960 \\
0.955\end{array}\right)$ & $0.014\left(\begin{array}{l}0.763 \\
0.719\end{array}\right)$ & $0.001\left(\begin{array}{l}0.996 \\
0.994\end{array}\right)$ & $0.007\left(\begin{array}{l}0.950 \\
0.930\end{array}\right)$ \\
\hline NGC4839 & $0.007\left(\begin{array}{l}0.943 \\
0.922\end{array}\right)$ & $0.002\left(\begin{array}{l}0.960 \\
0.955\end{array}\right)$ & $0.014\left(\begin{array}{l}0.763 \\
0.719\end{array}\right)$ & $0.001\left(\begin{array}{l}0.996 \\
0.994\end{array}\right)$ & $0.007\left(\begin{array}{l}0.950 \\
0.930\end{array}\right)$ \\
\hline NGC4873 & $0.002\left(\begin{array}{l}1.020 \\
1.013\end{array}\right)$ & $0.000(4)\left(\begin{array}{l}1.006 \\
1.005\end{array}\right)$ & $0.004\left(\begin{array}{l}1.076 \\
1.065\end{array}\right)$ & $0.000(2)\left(\begin{array}{l}1.001(1) \\
1.000(6)\end{array}\right)$ & $0.002\left(\begin{array}{l}1.017 \\
1.011\end{array}\right)$ \\
\hline
\end{tabular}

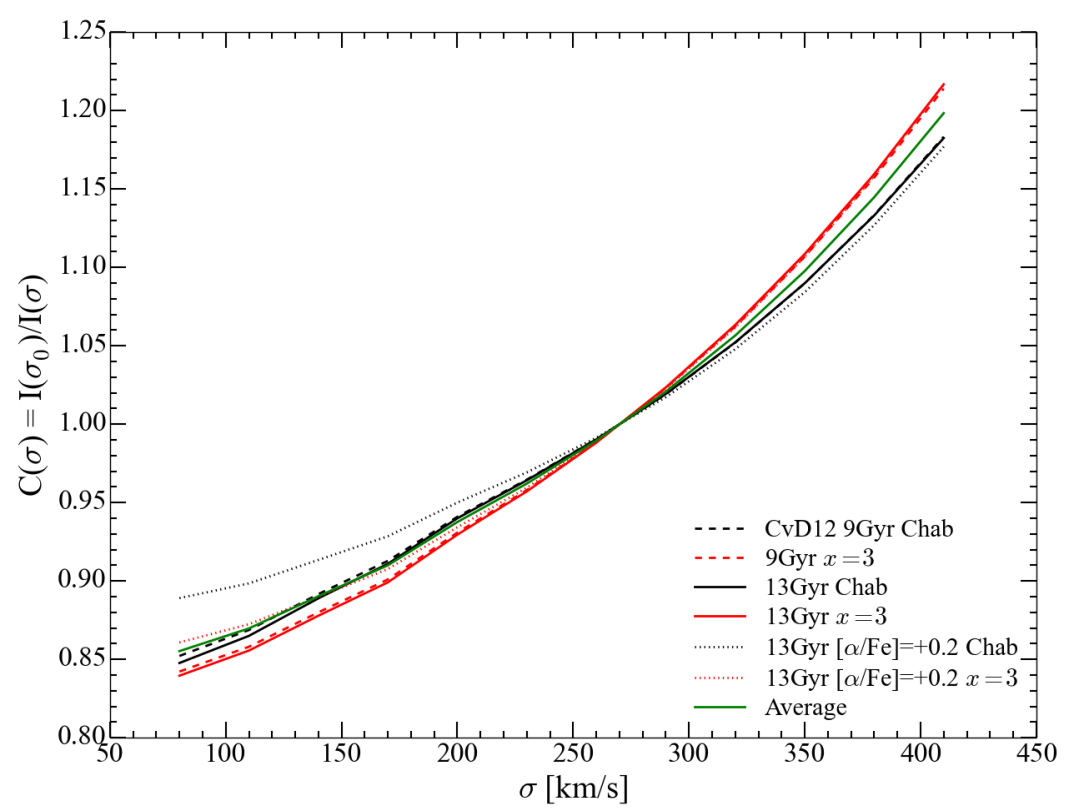

Figure A1. Plot showing the correction factor $C(\sigma)$ as a function of velocity dispersion $\sigma$ for the FeH index from a subset of CvD12 SSP spectra as shown in the legend. The correction factor is normalised to be unity at the intrinsic velocity dispersion of the galaxy and then the intrinsic galaxy index measurement is divided by the average value at the 'correcting' dispersion, for which in this paper we use $\sigma=200 \mathrm{~km} \mathrm{~s}^{-1}$. This plot shows the curves normalised at a value of $\sigma=270 \mathrm{~km} \mathrm{~s}^{-1}$, which then gives an value of $C\left(\sigma_{200}\right)=0.93$.

gle sky region over the FeH feature and pseudo-continuum definitions (shaded regions: shown at the redshift for each $\mathrm{BCG}$ ). Only scaling a single sky region across the $\mathrm{FeH}$ feature stops any differential gradient being introduced that can alter the resulting feature measurement on the order of tens of per cent. The right hand plots show the spectra corrected by the Sérsic profile fitting method as a comparison. NGC4889 shows consistent spectra at all radii. NGC4874 and NGC4839 have much more prominent sky lines across the FeH feature due to the increased redshift. The global spectra for NGC4839 shows a large systematic difference between the multi- and single- sky region PPXF correction, with the multi-region spectrum showing a much deeper feature. Thus, we cannot be certain whether the deeper FeH feature measured for NGC4839 is real or a result of the reduction process.

Fig. B3 shows our measurements of the $\mathrm{FeH}$ index for each BCG using the spectra from our three separate data reduction processes discussed in Section 2.3.2:

(a) telluric corrected spectra sky-subtracted using PPXF (multiple sky regions: green diamonds), (b) throughput corrected spectra sky-subtracted using PPXF (multiple sky regions: black circles, single sky region: red squares),

(c) throughput corrected spectra sky-subtracted using O-S spatial fitting (blue triangles).

For NGC4889 all measurements are in good agreement showing weak $\mathrm{FeH}$ at all radii. For NGC4874 the telluric corrected spectrum results in a much deeper $\mathrm{FeH}$ strength compared with the telluric continuum fitting method. This is caused by systematics in removing the Paschen features from the telluric spectrum artificially deepening the FeH feature. We therefore caution against performing telluric correction when $\mathrm{FeH}$ is redshifted long-ward of around $1 \mu \mathrm{m}$, as it moves into a region of negligible telluric absorption so any correction can introduce excess residuals. NGC4839 shows a large range of $\mathrm{FeH}$ strengths from the different sky subtraction procedures. The multiple sky region PPXF subtraction produces systematically deeper $\mathrm{FeH}$ features compared to the single sky region spectra. From Fig. B2 we see that $\mathrm{FeH}$ is redshifted into a prominent blend of sky lines between $1.015-1.025 \AA$, which is challenging to remove, and 
is therefore very difficult to measure cleanly. This causes the large systematic uncertainty on our $\mathrm{FeH}$ measurements for NGC4839, and also presents a limitation of the PPXF subtraction routine, justifying our use of an independent subtraction method.

\section{APPENDIX C: DERIVING IMF SLOPES AND CAVEATS}

To derive the IMF slopes we use the age-IMF grid of SSPs from the CvD12 models, covering 3-13.5 Gyr and bottomlight to bottom-heavy $x=3$ IMFs. Using the age of each galaxy inferred from Fig. 9 we interpolate the SSP grid and find a set of SSPs for the range of IMFs at a given age. We then use the varying element and $\alpha$-enhancement SSPs to derive response factors to apply to the varying IMF SSPs for variations in $[\alpha / \mathrm{Fe}],[\mathrm{Fe} / \mathrm{H}]$ and $[\mathrm{Na} / \mathrm{Fe}]$. By measuring the FeH index for each SSP (corrected for metallicity, $\alpha$ - and Na-enhancement), we compare the measured global value of $\mathrm{FeH}$ for each galaxy to the range of $\mathrm{FeH}$ values from the varying IMF SSPs. We propagate the fractional uncertainty in the FeH measurement through to the uncertainty in IMF slope as a first-order error approximation. We approximate a Chabrier IMF using a unimodal slope of $x=1.8$ and a bottom-light IMF using $x=1.6$ based upon matching the $(M / L)_{R}$ with that of the V15 unimodal, solar metallicity, 12.6 Gyr SSPs.

When deriving our IMF slopes as shown in Fig. 12 we apply response functions to the SSPs to account for variations in $[\alpha / \mathrm{Fe}],[\mathrm{Fe} / \mathrm{H}]$ and $[\mathrm{Na} / \mathrm{Fe}]$. The $\mathrm{CvD} 12$ models do not fully explore variations in total metallicity $[\mathrm{Z} / \mathrm{H}]$ so the corrections we apply to the SSP spectra (before measuring FeH to derive the IMFs as shown in Fig. 12), to account for variations in $[\alpha / \mathrm{Fe}],[\mathrm{Fe} / \mathrm{H}]$ and $[\mathrm{Na} / \mathrm{Fe}]$, are approximations. In Fig. C1 we show simple derivations of the IMF slope but without applying response functions to the SSPs to account for the stellar populations parameters. The results are fully consistent between the two plots showing that the variations in SSP parameters only yield second order effects. For example, increased metallicity, which enhances the $\mathrm{FeH}$ index strength, is offset by enhanced $[\mathrm{Na} / \mathrm{Fe}]$, which decreases the FeH strength in the CvD12 models. A comprehensive set of SPS models that allows for changes in the key parameters of $[\mathrm{Z} / \mathrm{H}],[\alpha / \mathrm{Fe}]$, individual element abundances (especially sodium), and covers the $\mathrm{FeH}$ wavelength region will help provide more accurate constraints on the IMF in ETGs.

This paper has been typeset from a $\mathrm{T}_{\mathrm{E}} \mathrm{X} / \mathrm{L} \mathrm{T}_{\mathrm{E}} \mathrm{X}$ file prepared by the author. 

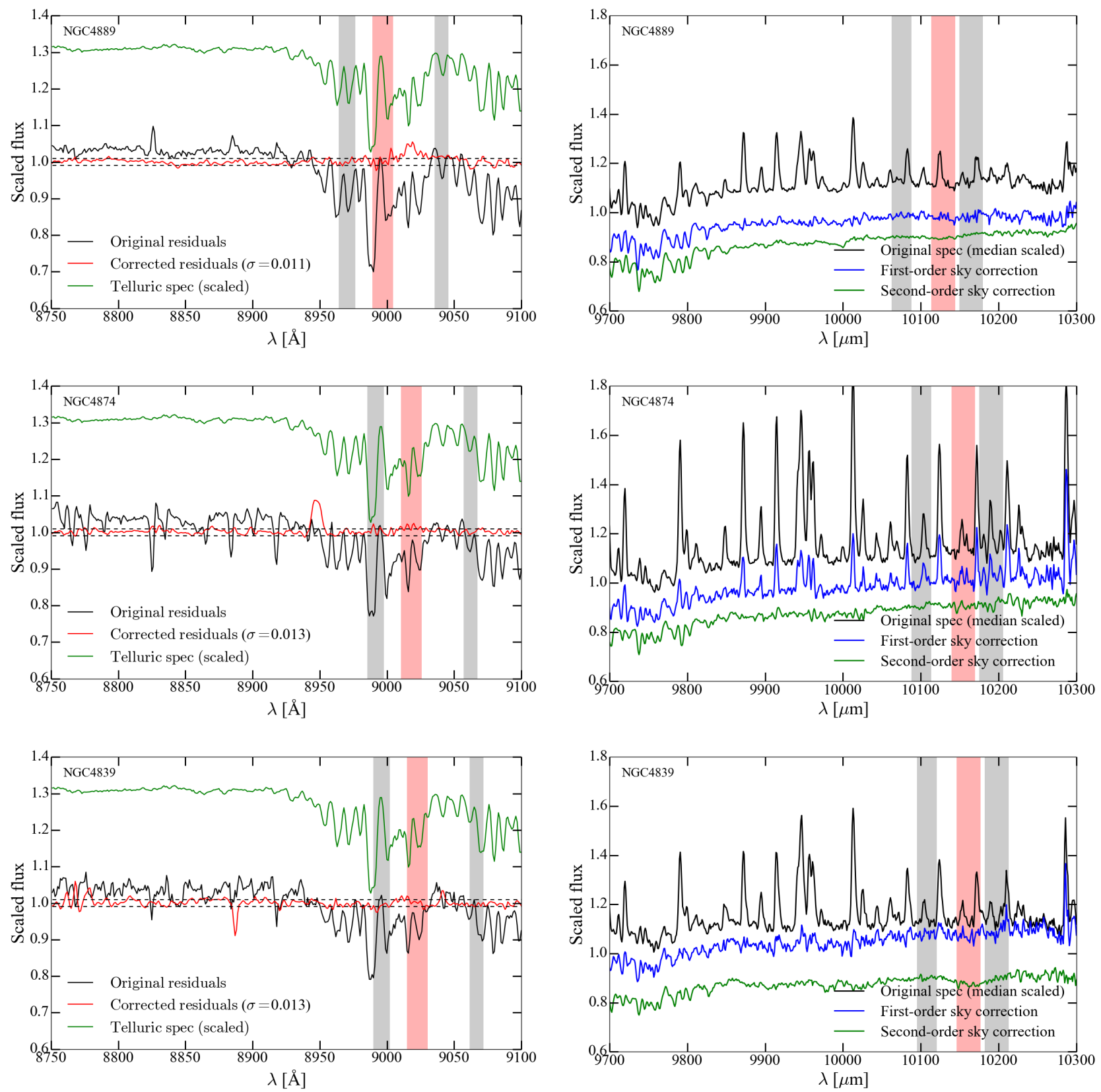

Figure B1. Plots showing telluric correction (left) and first- and second-order sky subtraction (right) for each BCG. The top two panels show NGC4889, the middle two show NGC4874 and the bottom two show NGC4839. The left-hand subplots show the telluric correction around MgI. The black line shows the ratio between original spectrum and kinematic template (assuming this is the intrinsic 'telluric free' spectrum), the red line shows the same ratio with telluric division, and the green line shows the telluric spectrum (arbitrarily scaled to fit onto plot). The position of the MgI feature and pseudo-continuum is shown by the shaded regions. The right-hand subplots show a comparison of the first- and second-order sky subtractions around the FeH feature. The first-order subtraction is achieved using a static sky data cube of the same size as the science cube (with separate cubes correctly shifted as to match spaxel positions). The second-order correction is performed using PPXF and removes the residuals at the level of a few percent left over from the first-order subtraction. The spectra are scaled by the median values and shifted vertically in the $y$-axis for clear presentation. 

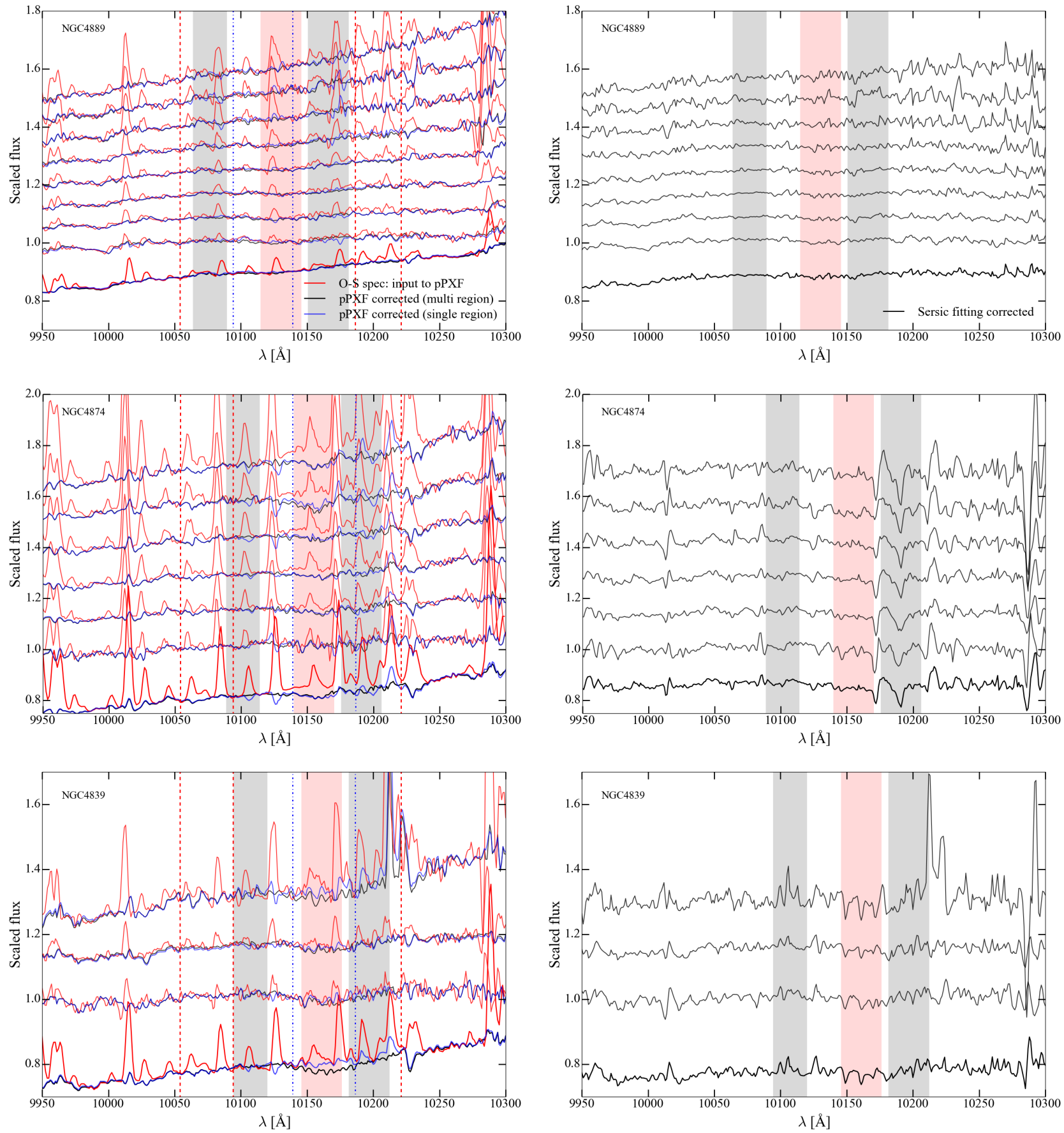

Figure B2. Plots showing the global (bold lines at bottom) and radial spectra (increasing radial distance bottom to top) around FeH for each BCG, before and after the two second-order sky subtraction routines: the PPXF method on the left hand side, and the Sérsic profile fitting on the right hand side. The top two panels show NGC4889, the middle two show NGC4874 and the bottom two show NGC4839. On the left hand panels, the input spectra to PPXF (red lines) are compared to the corrected spectra in black and blue. The sky regions that are scaled by PPXF to fit the sky lines in the science spectra are shown by the vertical lines. The black line output spectra are the results when scaling all sky regions shown by the red and blue vertical lines. The blue output spectra are the results when removing the sky regions shown by the vertical blue dotted lines and only scaling a single sky region over the FeH feature and pseudo-continuum definitions (shaded regions: shown at the redshift for each BCG). The right hand plots show the spectra corrected by the Sérsic profile fitting method as a comparison. NGC4889 shows consistent spectra at all radii. NGC4874 and NGC4839 have much more prominent sky lines across the $\mathrm{FeH}$ feature due to the increased redshift. The global spectra for NGC4839 shows a large systematic difference between the multi- and single- sky region PPXF correction, with the multi-region spectrum showing a much deeper feature. 


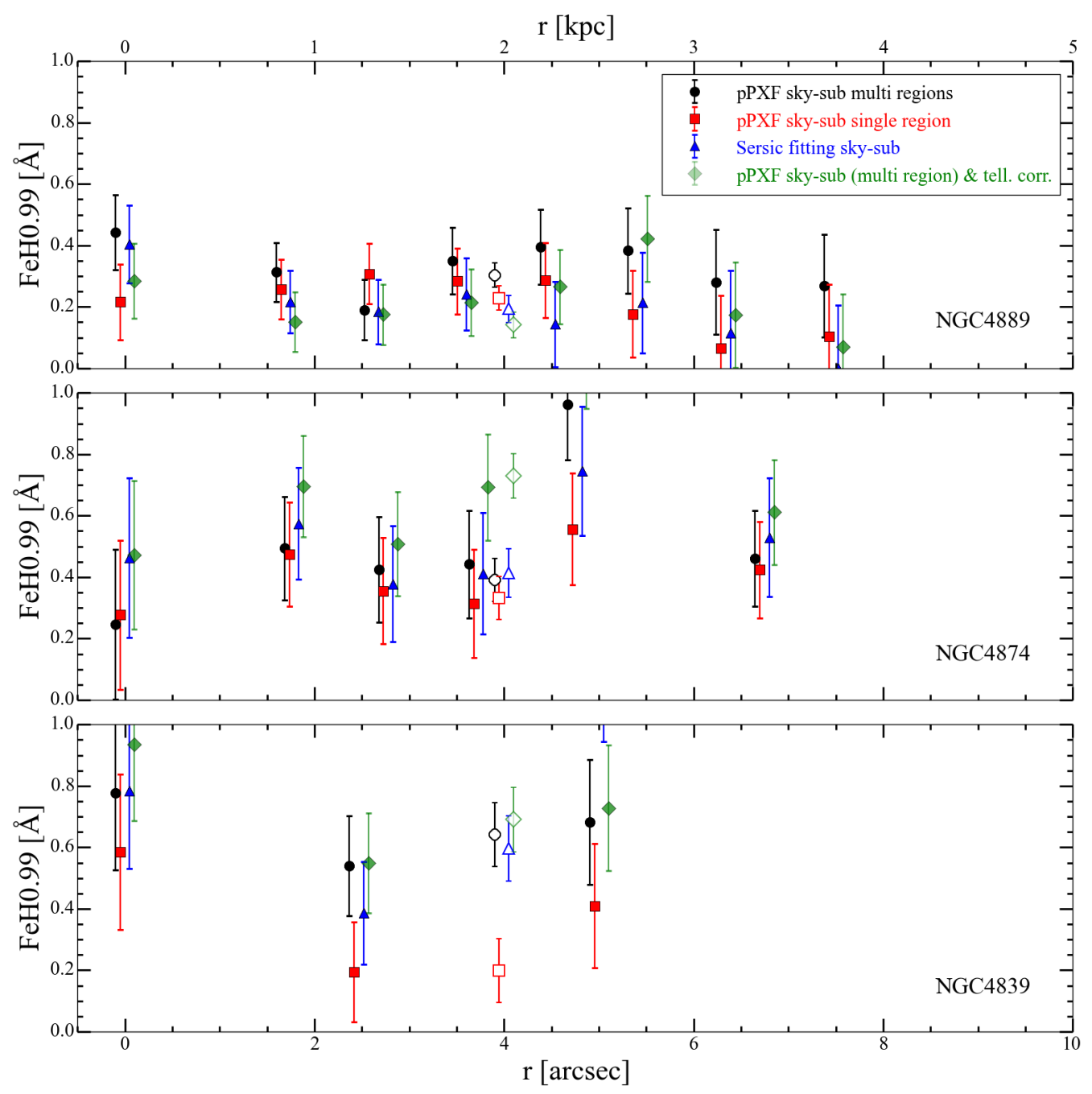

Figure B3. Plot showing radial measurements of FeH for each BCG, at a common resolution of $\sigma=200 \mathrm{~km} \mathrm{~s}^{-1}$, after the different sky-subtraction and telluric correction procedures. The sub-panels are labelled for each galaxy with NGC4889 at the top, NGC4874 in the middle and NGC4839 at the bottom. In each subplot, the black circles show measurements from spectra corrected by telluric polynomial fit division, and sky subtracted using PPXF with multiple sky regions across the FeH feature (shown in Fig. B2); the red squares are the same as the black circles but with only a single sky region across the FeH feature in the PPXF sky subtraction; the blue triangles show the measurements from spectra corrected by telluric polynomial fit division, and sky subtracted using the Sérsic fitting procedure in Section 2.3.2; the green diamonds show measurements from spectra corrected using a telluric spectrum and sky subtracted using PPXF with multiple sky regions across the FeH feature. 


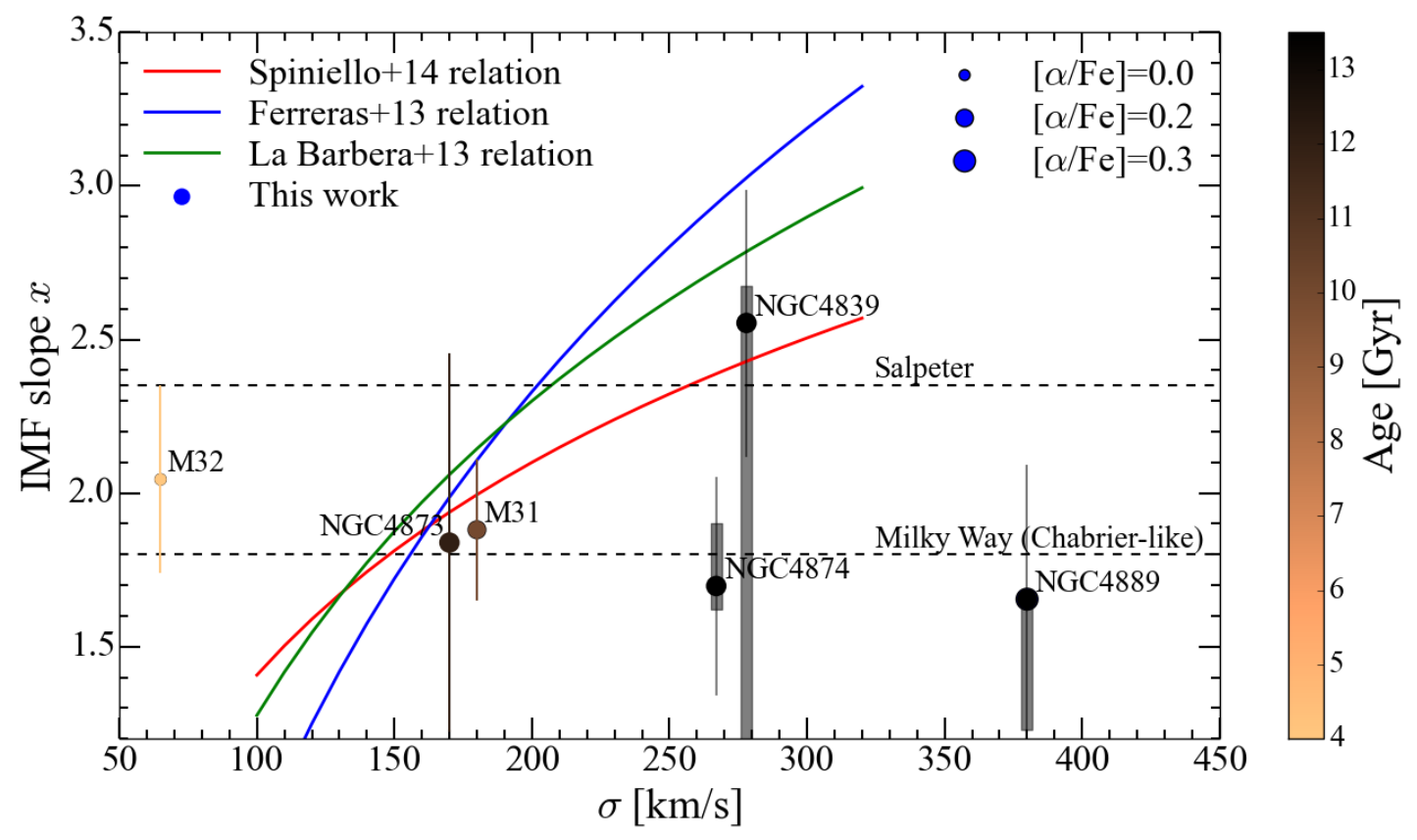

Figure C1. Same as Fig. 12 but without response functions applied when deriving the IMF slope: Derived IMF slope $x$ against central velocity dispersion for our sample of Coma galaxies, as well as M32 and M31 from Zieleniewski et al. (2015). The IMF slope has been derived from our global $\mathrm{FeH}$ measurements. For our data, the colour of each point indicates the age and the marker size indicates the level of $[\alpha / \mathrm{Fe}]$ enhancement for each galaxy. The coloured lines show unimodal IMF- $\sigma_{*}$ relations from the previous works of Ferreras et al. (2013); La Barbera et al. (2013) and Spiniello et al. (2014), derived using central 2.7" SDSS spectra and several optical/NIR features, including $\mathrm{NaD} / \mathrm{NaI}$ but excluding $\mathrm{FeH}$. The dashed horizontal lines show the Salpeter and Milky-Way (Chabrier-like) slopes. Our results show consistency with a universal Chabrier IMF across a large range of velocity dispersions, with the exception of the single galaxy NGC4839, for which we see possible evidence of an IMF slightly heavier than Salpeter, albeit with a very large systematic uncertainty. 